Western University

Scholarship@Western

Digitized Theses

Digitized Special Collections

1970

\title{
A Tauberian Theorem For Borel-type Methods Of Summability
}

Irvine Joseph Robinson

Follow this and additional works at: https://ir.lib.uwo.ca/digitizedtheses

\section{Recommended Citation}

Robinson, Irvine Joseph, "A Tauberian Theorem For Borel-type Methods Of Summability" (1970). Digitized Theses. 450.

https://ir.lib.uwo.ca/digitizedtheses/450

This Dissertation is brought to you for free and open access by the Digitized Special Collections at Scholarship@Western. It has been accepted for inclusion in Digitized Theses by an authorized administrator of Scholarship@Western. For more information, please contact tadam@uwo.ca,

wlswadmin@uwo.ca. 
The author of this thesis has granted The University of Western Ontario a non-exclusive license to reproduce and distribute copies of this thesis to users of Western Libraries. Copyright remains with the author.

Electronic theses and dissertations available in The University of Western Ontario's institutional repository (Scholarship@Western) are solely for the purpose of private study and research. They may not be copied or reproduced, except as permitted by copyright laws, without written authority of the copyright owner. Any commercial use or publication is strictly prohibited.

The original copyright license attesting to these terms and signed by the author of this thesis may be found in the original print version of the thesis, held by Western Libraries.

The thesis approval page signed by the examining committee may also be found in the original print version of the thesis held in Western Libraries.

Please contact Western Libraries for further information:

E-mail: libadmin@uwo.ca

Telephone: (519) 661-2111 Ext. 84796

Web site: http://www.lib.uwo.ca/ 
A TAUBERIAN THEOREM FOR BOREL-TYPE METEODS OF SUMAABILITI

By

Irvine Joseph Warrington Robinson

Department of Mathematics

Submitted in partial fulfillment

of the requirements for the degree of

Doctor of Philosophy

Faculty of Graduate Studies

The University of Western Ontario

London, Canada.

September, 1969

Irvine Joseph Warringtor Robinson 1969 


\section{ABSTRACT}

A series of real or complex numbers $\sum_{n=0}^{\infty} a_{n}$ is said to be summable $(B, \alpha, \beta)$ to $A$ if

$\alpha e^{-x} \sum_{n=N}^{\infty} A_{n} \frac{x^{\alpha n+\beta-1}}{\Gamma(\alpha n+\beta)} \rightarrow A$ as $x+\infty$

where $\alpha>0, \beta$ is real, and $N$ is a non-negative integer such that $\alpha N+\beta>0$. The standard Borel exponential. method of summability $B$ is just the special case $(B, I, I)$.

The main result of this thesis is the following "O" Tauberian Theorem for summability $(B, \alpha, \beta)$ :

If $a_{n}=O\left(n^{-1 / 2}\right) \quad\left(\underline{i}\right.$ e. the sequence $\left\{\sqrt{n} a_{n}: n=1,2,3, \ldots\right\}$ is bounded), and $\sum_{n=0}^{\infty} a_{n}=A(B, \alpha, \beta)$, then $\sum_{n=0}^{\infty} a_{n}=A$.

A known method of summability $(e, c)$ is considered and it is shown that summability $(B, \alpha, \beta)$ is equivalent to summability $(e, \alpha / 2)$ if $a_{n}=o(I)$. It is then shown that if $0<d<c$, summability $(e, c)$ and $a_{n}=o(1)$ 


$$
\begin{aligned}
& \text { together imply summability }(e, d) \text {. This is done by } \\
& \text { considering another known method of sinmability }(\gamma, k) \text {, } \\
& \text { showing that summability }(\gamma, k) \text { implies summability }(\gamma, l) \\
& \text { if } 0<\ell<k \text {, and that, if } a_{n}=o(I) \text {, summability } \\
& (\gamma, k) \text { is equivalent to summability }(e, c) \text { where } c=\frac{k}{2(1-k)} \text {. } \\
& \text { Summability }(B, \alpha, \beta) \text { and } a_{n}=0\left(n^{-1 / 2}\right) \text { are shown to } \\
& \text { imply that } A_{n}=O(I) \text {. Vitali's Theorem is then used } \\
& \text { to show that if } A_{n}=0(I) \text {, summability }(B, \alpha, \beta) \text { implies } \\
& \text { summability }(e, c) \text { for all positive } c \text {. The desired "o" } \\
& \text { Tauberian Theorem is then deduced. }
\end{aligned}
$$




\section{ACKNOWLEDGEMENTS}

I am deeply indebted to Dr. D. Borwein for his kindness, encouragement and academic guidance.

I would also like to express my thanks to my wife who typed the first draft of this thesis, and to Miss Christine McCulloch who typed the final draft. 


\section{TABLE OF CONTENTS}

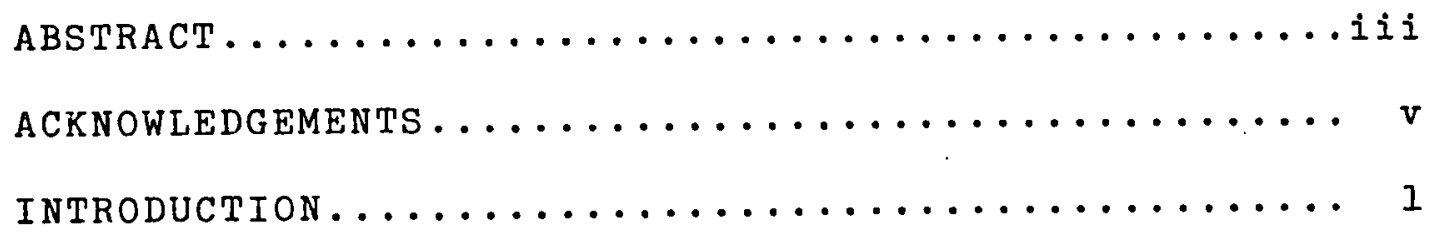

CHAPTER 1. Known results and some preliminary

Lemmas..................... 6

CHAPTER 2. Some relations between certain sums and integrals........................ 14

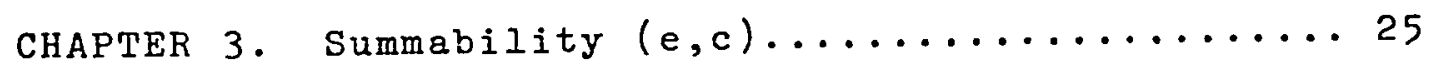

CHAPTER 4. Summability $(\gamma, k) \ldots \ldots \ldots \ldots \ldots \ldots \ldots \ldots \ldots$

CHAPTER 5. Vitali's Theorem................... 64

CHAPTER 6. The "O" Tauberian Theorem for

summability $(B, \alpha, \beta) \ldots \ldots \ldots \ldots \ldots \ldots 68$

REFERENCES......................... 79

$\operatorname{vita} \ldots \ldots \ldots \ldots \ldots \ldots \ldots \ldots \ldots \ldots \ldots \ldots \ldots$ 


\section{INTRODUCTION}

\section{(1) Notation.}

Let $\sum_{n=0}^{\infty} a_{n}$ be a series of complex (or real) numbers.

Let $A_{n}$ denote the partial sum $a_{0}+\ldots+a_{n}$ of the series if $n \geq 0$ and let $A_{n}=0$ if $n<0$.

The symbol $[x]$ denotes the greatest integer $\leq x$.

\section{(2) Definitions and discussion.}

If a given method of summability $T$ assigns the

"sum" A to the series $\sum_{n=0}^{\infty} a_{n}$, we say that $\sum_{n=0}^{\infty} a_{n}$ is

summable $T$ to $A$ and write $\sum_{n=0}^{\infty} a_{n}=A(T)$ or $A_{2} \rightarrow A(T)$.

The method $T$ is said to be regular if it sums every convergent series to its ordinary sum.

Now suppose that $\alpha>0, B$ is real, and $N$ is a non-negative integer such that $\alpha \mathbb{N}+\beta>0$. We say that the series $\sum_{n=0}^{\infty} a_{n}$ is summable $(\bar{B}, \alpha, \beta)$ to $A$ and writo $\sum_{n=0}^{\infty} \varepsilon_{n}=A(B, \alpha, \beta)$ if $\alpha e^{-x} \sum_{n=N}^{\infty} \frac{A_{n} x^{\alpha n+\beta-1}}{\Gamma(\alpha n+\beta)} \quad+A$ as $x \rightarrow \infty$. 
Since $e^{-x} \frac{A_{k} x^{\alpha k+\beta-1}}{\Gamma(\alpha k+\beta)} \quad \rightarrow 0$ as $x \rightarrow \infty$, it is clear that the actual choice of $N$ is immaterial.

The method $(B, I, I)$ is the standard Borel exponential method of summability $B$.

For the definition and early investigation of summability $(B, \alpha, \beta)$ see Borwein [2], [3], and [4], Good [6], Hardy [7], and Wxodarski [14]. For Borel summability B see Hardy [8]. The method $(B, \alpha, \beta)$ is equivalent to a special case of a very general type of summability method, usually known as a $J$ method or integral function method (although entire function method would perhaps be more apt). Let $J(z)=\sum_{n=0}^{\infty} J_{n} z^{n} \quad, J_{n} \geq 0$, where $J(z)$ is an entire function (i.e. analytic in the entire complex plane) but not a polynomial. Then $\sum_{n=0}^{\infty} a_{n}=A(J)$ if $\frac{\sum_{n=0}^{\infty} J_{n} A_{n} x^{n}}{J(x)} \rightarrow A$ as $x \rightarrow \infty$ Since $\sum_{n=N}^{\infty} \frac{x^{\alpha n+\beta-1}}{\Gamma(\alpha n+\beta)} \sim \frac{e^{x}}{\alpha}$ as $x \rightarrow \infty$ (see Borwein [4], p. 130, with $\delta=\beta-1)$, it follows that the $(B, \alpha, \hat{B})$ method is equivalent to the $J$ method defined by setting 


$$
J_{n}=\left\{\begin{array}{cl}
\frac{1}{\Gamma(\alpha n+\beta)} & , n \geq N \\
0 & , 0 \leq n<N .
\end{array}\right.
$$

(substituting $y$ for $x^{2}$ and then writing $x$ ior $y$ gives $\frac{1}{J(x)} \sim \alpha x^{(B-1) / \alpha} e^{-x^{1 / \alpha}}$. Then setting $u=x^{1 / \alpha}$, it follows that $\frac{\sum_{n=0}^{\infty} J_{n} A_{n} x^{n}}{J(x)} \sim \alpha e^{-u} \sum_{n=N}^{\infty} \frac{A_{n} u^{\alpha n+\beta-1}}{\Gamma(\alpha n+\beta)}$.)

Since $J$ methods are known to be regular (see Hardy [8], Theorem 33]), summability $(B, \alpha, \beta)$ is a regular method.

The main object in this thesis is to prove the following theorem.

If $\sum_{n=0}^{\infty} a_{n}=A(B, \alpha, \beta)$ and $a_{n}=0\left(n^{-1 / 2}\right)$ then $\sum_{n=0}^{\infty} a_{n}=A$. (This is Theorem (6.7) of the thesis; see the end of this introduction for the method of numbering.)

This is an example of a "O" Tauberian Theorem in that we have the summability of a series by a regular method together. with a "O" condition (the Tauberian condition) implying its convergence.

The corresponding theorem for Borel summability $B$ was first proved by Hardy and istiliewood [9]. It is the main result of chapter nine of Hardy's book [8]. That summability $(B, \alpha, \beta)$ of $\sum_{n=0}^{\infty} a_{n}$ and $a_{n}=0\left(n^{-1 / 2}\right.$; 
iogether imply the convergence of the series is a special case of Borwein [5]. (This is the "o" Tauberian Theorem for summability $(B, \alpha, \beta)$.)

Borwein [4, (III) and (IV)] has proved that:

If $\mathrm{J}(\mathrm{z})=\sum_{\mathrm{n}=\mathrm{N}}^{\infty} \frac{\mathrm{z}^{\mathrm{n}}}{\mathrm{h}(\mathrm{n})}$ where $\mathrm{h}(\mathrm{z})$ is an analytic

function of $\mathrm{z}=\mathrm{x}+$ iy in the region $\mathrm{x}>\mathrm{x}_{0}$, such that

(i) when $x>x_{0}$ and $|z|$ is Zarge.

$$
\mathrm{h}(\mathrm{z})=\mathrm{z}^{\alpha \mathrm{z}+\beta} \mathrm{e}^{\gamma \mathrm{z}}\left\{\mathrm{c}+0\left(\frac{\mathrm{l}}{|\mathrm{z}|}\right)\right\} \text { where } \mathrm{c}>0, \alpha>0
$$

$B$ and $r$ are real,

and

(ii) $h(x)$ is real for $x>x_{0}$,

then

$\sum_{n=0}^{\infty} a_{n}=A(J)$ if and only if $\sum_{n=0}^{\infty} a_{n}=A(B, \alpha, \beta+1 / 2)$.

In particular, taking $\mathrm{h}(\mathrm{z})=\{\Gamma(\mathrm{az}+\mathrm{b})\}^{c}(\mathrm{z}+\mathrm{p})^{\mathrm{qz}+\mathrm{r}}$

where $\mathrm{b}, \mathrm{c}, \mathrm{p}, \mathrm{q}$, and $\mathrm{r}$ are real, $\mathrm{a}>0$ and $\mathrm{ac}+\mathrm{q}>0$, so that

$$
\begin{aligned}
& J(z)=\sum_{n=\mathbb{N}}^{\infty} \frac{z^{n}}{\{\Gamma(a n+b)\}^{c}(n+p)^{q n+r}} \\
& \sum_{n=0}^{\infty} a_{n}=A(J) \text { if and oniy if } \\
& \sum_{n=0}^{\infty} a_{n}=A(B, a c+q, b c+r-c / 2+I / 2) .
\end{aligned}
$$


It follows that Theorem $(6.7)$ is in fact a Tauberian Theorem for quite a wide class of summability methods. Theorem $(6.7)$ remains true if $\sum_{n=0}^{\infty} a_{n}=A(B, \alpha, \beta)$

is replaced by $\sum_{n=0}^{\infty} a_{n}=A\left(B^{\prime}, \alpha, \beta\right)$, by which it is meant that, as $y+\infty, \int_{0}^{y} e^{-x} d x \sum_{n=N}^{\infty} \frac{a_{n} x^{\alpha n+\beta-1}}{\Gamma(\alpha n+\beta)}+A-A_{N-1}$ This is a consequence of the following known result (see Borwein [3, Theorem 2]):

$\sum_{n=0}^{\infty} a_{n}=A(B, \alpha, \beta+1)$ if and only if $\sum_{n=0}^{\infty} a_{n}=A\left(B^{\prime}, \alpha, \beta\right)$.

(3) Method of numbering.

The thesis contains this introduction and six chapters. Theorems, lemmas, corollaries, and equations to which we will later refer are numbered consecutively within each chapter. The number is placed first and is in the margin for asse of reference. Thus in chapter three, our first result is listed as (3.1) THEOREM and would be referred to as (3.1) or Theorem (3.1). 


\section{CHAPTER 1}

\section{KNOWN RESULTS AND SOME PRELIMINARY LEMMAS}

We first state some results of D. Borwein [5] which are needed in later chapters.

(1.1) If $\rho \geq-1 / 2, a_{n}=o\left(n^{p}\right)$ and $\sum_{n=0}^{\infty} a_{n}=0(B, \alpha, \beta)$, then $A_{n}=o\left(n^{\rho+1 / 2}\right)$.

This is Lemma 5 of Borwein [5] with $k=0, \mu=1$, and $\lambda=\rho$. The result we need follows immediately:

(1.2) If $\rho \geq-1 / 2, a_{n}=o\left(n^{\rho}\right)$ and $\sum_{n=0}^{\infty} a_{n}=A(B, \alpha, \beta)$, then $A_{n}=o\left(n^{\rho+1 / 2}\right)$.

$$
\text { Let } \mathrm{x}>0, \mathrm{~h}=\mathrm{n}-\mathrm{x} / \alpha, I / 2<\zeta<2 / 3 \text {, and }
$$

$0<n<2 \zeta-1$.

Then

$$
\begin{aligned}
& \text { (1.3) } e^{-x} \sum_{|h|>x} x^{\frac{x^{\alpha n+B-1}}{\Gamma(\alpha n+\beta)}}=0\left(e^{-x^{n}}\right) \\
& (\text { Lemma 2, part (d) of Borwein [5]) }
\end{aligned}
$$


Chap. 1

and

(1.4) $e^{-x} \frac{x^{\alpha n+\beta-1}}{\Gamma(\alpha n+\beta)}=\frac{1}{\sqrt{2 \pi x}} e^{-\alpha^{2} h^{2} / 2 x}\left\{1+0\left(x^{3 \zeta-2}\right)\right\}$

if $|\mathrm{h}| \leq \mathrm{x}^{5}$

(Lemma 2, part (e) of Borwein [5]).

In fact we need the slightly sharper estimate:

(1.5) $e^{-x} \frac{x^{\alpha n+\beta-1}}{\Gamma(\alpha n+\beta)}=\frac{1}{\sqrt{2 \pi x}} e^{-\alpha^{2} h^{2} / 2 x} \quad\left\{1+0\left(\frac{|h|+1}{x}\right)+0\left(\frac{|h|^{3}}{x^{2}}\right)\right\}$

if $|\mathrm{h}| \leq \mathrm{x}^{5}$

This would have been obtained if Borwein had not used $\frac{\lfloor h \mid+1}{x}=0\left(x^{3 \zeta-2}\right)$ and $\frac{|h|^{3}}{x^{2}}=0\left(x^{3 \zeta-2}\right)$ in simplifying near the end of his proof. In (1.5) we write $0\left(\frac{|h|+1}{x}\right)$ instead of $0\left(\frac{|h|}{x}\right)$ in order to include the case $h=0$.

It follows from Stirling's Theorem that

(1.6) $\Gamma(\alpha n+\beta)=(2 \pi)^{1 / 2} e^{\alpha n}(\alpha n)^{\alpha n+\beta-1 / 2}\{1+0(1 / n)\}$

(See Higher Transcendental functions [T, formula 3 , I.I8, p. 47, vol. I].)

It follows that $\frac{(\alpha n)^{1 / 2} \Gamma(\alpha n+\beta-I / 2)}{\Gamma(\alpha n+\beta)} \sim 1$ and hence that $(1.7) \frac{n^{1 / 2}}{\Gamma(\alpha n+\beta)}=0\left(\frac{1}{\Gamma(\alpha n+\bar{\beta}-1 / 2)}\right)$. 
Chap. 1

The next four well known results are stated without proof and will be used without reference.

$x^{\lambda} e^{-p x^{a}}=o(1)$ as $x \rightarrow \infty$ for all real $\lambda, p>0, a>0$. For $n \geq 0:$

$$
\begin{aligned}
& n^{\lambda} e^{-\mu n}=0\left(e^{-\gamma n}\right) \text { if } 0<\gamma<\mu, \lambda \text { real. } \\
& e^{-\mu n}=0\left(e^{-n^{n}}\right) \text { if } \mu>0, n>0 . \\
& e^{-c n^{a}}=0\left(e^{-n^{b}}\right) \text { if } c>0 \text { and } 0<b<a .
\end{aligned}
$$

(1.8) LEMMA.

$$
\text { For } 0<u \leq H \quad, e^{O(u)}=1+O(u) \text {. }
$$

PROOF.

$$
\text { Let } v=O(u) \text { where } 0<u \leq H \text {. Then }|v| \leq K u \text { for }
$$
some constant $K$, and so

$\left|e^{v}-I\right|=\left|v \sum_{n=1}^{\infty} \frac{v^{n-1}}{n !}\right| \leq u\left\{K \sum_{n=1}^{\infty} \frac{(K H)^{n-1}}{n !}\right\}=c u$

for some constant $C$, since the series converges. The result follows.

(1.9) LEMMA.

$$
\text { For }|u| \leq r<1, \log (1+u)=u-u^{2} / 2+0\left(|u|^{3}\right) .
$$


Chap. 1

PROOF.

$\operatorname{For}|u|<2$,

$108(1+u)=\sum_{n=1}^{\infty}(-1)^{n-1} u^{n} / n=u-u^{2} / 2+u^{3} \sum_{n=0}^{\infty}(-1)^{n} \frac{u^{n}}{n+3}$,

and therefore

$$
\begin{aligned}
& \left|10 g(1+u)-\left(u-u^{2} / 2\right)\right| \leq|u|^{3} \sum_{n=0}^{\infty} \frac{|u|^{n}}{n+3} \\
& \leq|u|^{3} \sum_{n=0}^{\infty} \frac{r^{n}}{n+3}=c|u|^{3} \quad \text { for some constant } C,
\end{aligned}
$$

since the series converges. The result follows.

In the rest of this chapter some order relations

involving partial sums of the series $\sum_{n=0}^{\infty} a_{n}$, which will

be needed later, are stated and proved as lemmas.

We assume throughout that $1 / 2<\zeta<2 / 3,0<\mathrm{k}<1$, and $\mathrm{n}$ is a positive integer.

(1.10) LEMMA.

$$
\text { If } \lambda>0 \text { and } A_{n}=o\left(n^{\lambda}\right) \text { then } A_{j+h}=0\left((j+|h|)^{\lambda}\right)
$$

where $J$ is a positive integer. In particular this holds for either $\mathrm{j}=$ in or $\mathrm{j}=\mathrm{M}=[\mathrm{n} / \mathrm{k}]$.

PROOF .

Since $\Lambda_{n}=o\left(n^{\lambda}\right)$ there exists an $N_{0}$ such that if $j+h>N_{0}$ then $\left|\Lambda_{j+h}\right| \leq(j+h)^{\lambda} \leq(j+|h|)^{\lambda}$. Since there 
is only a finite number of integers between 0 and $N_{0}$,

there is a constant $K>0$ such that $\left|A_{j+h}\right| \leq K(j+|h|)^{\lambda}$

for $j+h=0,1,2, \ldots, N_{0}$. Thus $A_{j+h}=0(j+|h|)^{\lambda}$.

\section{(1.11) LENHÄA.}

$$
\text { If } \mathrm{a}_{\mathrm{n}}=\mathrm{o}(1) \text { and }|\mathrm{h}| \leq \mathrm{n}^{5} \text {; then, }
$$

for either $j=n$ or $j=M=[n / k]$,

$A_{j+h}-A_{j}=0(|h|)$ as $n+\infty$, uniformly for $|h| \leq n^{5}$.

(This is a special case of Hardy [8, Theorem 144] with

$\alpha=-1, \beta=1, \rho=0$ since for $n$ sufficiently large

$\mathrm{n}^{5} \leq \mathrm{n} / 2=\mathrm{Hn}$. Our case is easy and we prove it directly.)

PROOF.

$$
\text { Since } a_{n}=o(I) \text {, given } E>0 \text { there exists an } N_{0}
$$

such that if $r \geq N_{0}$ then $\left|a_{r}\right|<\varepsilon$. Thus

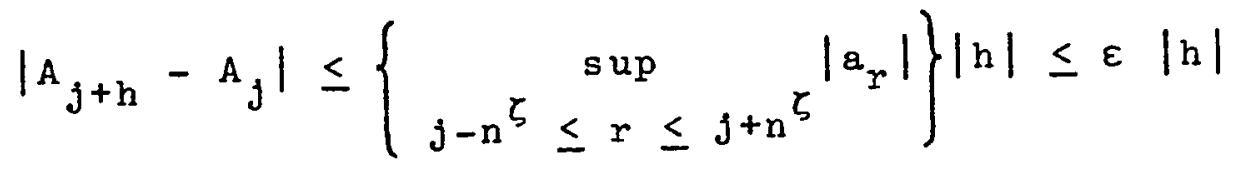

if $j-n^{\zeta} \geq N_{0}$.

Since for either $j=n$ or $j=M, j-n^{5}+\infty$ as $n \rightarrow \infty$,

it follows that $A_{j+h}-A_{j}=o(|h|)$ as $n \rightarrow \infty$, uniformly

for $|n| \leq n^{\zeta}$ 
Chap. I

11

(1.12) LEMMA.

$$
\text { If } \mathrm{A}_{\mathrm{n}}=\mathrm{o}\left(\mathrm{n}^{\mathrm{I} / 2}\right) \text { and }|\mathrm{n}| \leq\left(\alpha_{\mathrm{n}}\right)^{\overline{5}} \text {, then, }
$$

for either $j=n$ or $j=M=[n / k]$,

$$
A_{j+h}=o\left(j^{1 / 2}\right) \text { as } n \rightarrow \infty \text {, uniformly for }|\mathrm{h}| \leq(\alpha \mathrm{n})^{\zeta} \text {. }
$$

PROOF.

$$
\begin{aligned}
& \frac{|A j+h|}{j^{1 / 2}}=(1+h / j)^{1 / 2} \frac{\left|A_{j+h}\right|}{(j+h)^{1 / 2}} \\
& \leq\left(1+\frac{\left(\alpha_{n}\right)^{\zeta}}{j}\right)^{1 / 2} \frac{\left|A_{j+h}\right|}{(j+h)^{1 / 2}} \\
& \leq H \frac{\left|A_{j}+h\right|}{(j+h)^{I / 2}} \text { for some constant } H>0 \text {, } \\
& \text { since }\left(1+\frac{(\alpha n)^{\zeta}}{j}\right)^{1 / 2} \rightarrow 1 \text { as } n \rightarrow \infty \text { for either } j=n \\
& \text { or } j=[n / k] \text {. Because }|h| \leq(\alpha n)^{\zeta}, j+n \geq j-(\alpha n)^{\zeta} \text {. } \\
& \text { since for either } j=n \text { or } j=M, j-(\alpha n)^{\zeta} \rightarrow \infty \text { as } n \rightarrow \infty \text {, } \\
& \frac{\left|A_{j+h}\right|}{(j+h)^{I / 2}}=o(1) \text { as } n \rightarrow \infty \text {, uniformly for }|h| \leq(\alpha n)^{\zeta} \text {. } \\
& \text { Therefore } A_{j+h}=o\left(j^{1 / 2}\right) \text { as } n \rightarrow \infty \text {, uniformly for } \\
& |h| \leq(a n)^{\zeta} \quad(j=n \text { or } j=M)
\end{aligned}
$$


Chep. 1

(1.13) LEMMA.

$$
\text { If } \mathrm{A}_{\mathrm{n}}=\mathrm{o}\left(\mathrm{n}^{1 / 2}\right) \text { and }|\mathrm{h}|>\left(\alpha_{\mathrm{n}}\right)^{\zeta}>0 \text {, then, }
$$

for either $j=n$ or $j=M=[n / k], \quad A_{j+h}=0(|h|)$.

PROOF.

It follows from Lemma (1.10) with $\lambda=1 / 2$ that

$A_{M+h}=0\left\{(M+|h|)^{1 / 2}\right\}$. Since $M=[n / k]=O(n)$,

$M+|h|=O(n+|h|)$ and $(M+|h|)^{1 / 2}=0\left\{(n+|h|)^{1 / 2}\right\}$.

Thus in either case $A_{j+h}=0\left\{(n+|h|)^{1 / 2}\right\}$.

Now $1 / 2<\zeta<2 / 3$ and therefore $3 / 2<1 / \zeta<2$ and

$3 / 4<1 / 2 \zeta<1$

Since $|\mathrm{h}|>(\alpha \mathrm{n})^{\zeta}, \mathrm{n}=0\left(|\mathrm{~h}|^{1 / \zeta}\right)$.

Thus

$$
\mathrm{n}+|\mathrm{h}|=0\left(|\mathrm{~h}|^{1 / \zeta}\right)+|\mathrm{h}|=0\left(|\mathrm{~h}|^{1 / \zeta}\right)
$$

and

$$
(n+|h|)^{1 / 2}=0\left(|h|^{1 / 2 \zeta}\right)=0(|h|) .
$$

Therefore $A_{j+h}=0(|h|)$.

(1.14) LEMMAR.

$$
\begin{aligned}
\text { If } a_{n} & =0\left(n^{-1 / 2}\right) \text { then, for }|h| \leq n^{5}, \\
A_{n+h}-A_{n} & =0\left(\frac{|n|}{\sqrt{n}}\right) .
\end{aligned}
$$


Chap. 1

13

PROOF.

$$
\begin{aligned}
& \text { If } h>0 \text {, } \\
& \left|A_{n+h}-A_{n}\right| \leq\left|a_{n+1}\right|+\ldots+\left|a_{n+h}\right| \\
& \leq H\left(\frac{1}{\sqrt{n+1}}+\cdots+\frac{1}{\sqrt{n+h}}\right)<\frac{H h}{\sqrt{n}} . \\
& \text { If } h<0 \text {, } \\
& \left|A_{n+h}-A_{n}\right| \leq\left|a_{n+h+1}\right|+\ldots+\left|a_{n}\right| \\
& \leq H\left(\frac{1}{\sqrt{n+h+1}}+\cdots+\frac{1}{\sqrt{n}}\right) \leq \frac{H|h|}{\sqrt{n-|h|}} \\
& \leq \frac{H}{\left(1-n^{\zeta-1}\right)^{1 / 2}}\left(\frac{|n|}{\sqrt{n}}\right) \text {. } \\
& \text { Since }\left(1-n^{5-1}\right)^{1 / 2} \rightarrow 1 \text { as } n \rightarrow \infty \text { it follows that } \\
& \left|A_{n+h}-A_{n}\right| \leq K \frac{|h|}{\sqrt{n}} \text { for some constant } K>0 \text {. } \\
& \text { Therefore }\left|A_{n+h}-A_{n}\right|=0\left(\frac{|h|}{\sqrt{n}}\right) \text {. }
\end{aligned}
$$




\section{CHAPTER 2}

\section{SOME RELATIONS BETWEEN CERTAIIN SUMS AND INTEGRALS}

Our main concern in this chapter will be to establish estimates for certain types of sums in terms of integrals. These estimates will enable us, in later chapters, to replace (whenever it is convenient to do so) such a sum by the corresponding integral.

In our first two lemmas we evaluate the integrals which concern us. Throughout the chapter $c>0$ and $n$ is a positive integer.

(2.1) LEMMA.

$$
\int_{0}^{\infty} e^{-c t^{2} / n} t^{\lambda} d t=\left\{\begin{array}{ll}
(1 / 2)(\pi n / c)^{1 / 2} & , \lambda=0 \\
n / 2 c & , \lambda=1 \\
n^{2} / 2 c^{2} & , \lambda=3
\end{array} .\right.
$$

PROOF.

$$
\text { Setting } y=(c / n) t^{2}, t=(n / c)^{1 / 2} y^{1 / 2},
$$

$d t=(\mathrm{n} / \mathrm{c})^{1 / 2}(1 / 2) y^{-1 / 2} d y$, the integral becomes

$\frac{1}{2}(n / c)^{\frac{\lambda+1}{2}} \int_{0}^{\infty} c^{-y} y^{\frac{\lambda-1}{2}} d y=\frac{1}{2}(n / c)^{\frac{\lambda+1}{2}} \Gamma\left(\frac{\lambda+1}{2}\right)$.

The result follows since $\Gamma(1 / 2)=\sqrt{\pi}$ while $\Gamma(1)=\Gamma(2)=1$. 
Cliap. 2

(2.2) LEMMA.

$$
\int_{(\alpha n)^{\zeta}}^{\infty} e^{-c t^{2} / n} t a t=(n / 2 c) e^{-c \alpha^{2 \zeta} n^{2 \zeta-1}}, 1 / 2<\zeta<2 / 3 .
$$

PROOOF.

$$
\text { set } u^{2}=c t^{2} / n, \quad t=(n / c)^{1 / 2} u, d t=(n / c)^{1 / 2} d u \text {, }
$$

and the integral becomes

$$
\begin{aligned}
c^{1 / 2} \int_{\alpha^{\zeta}{ }^{5-1 / 2}}^{\infty} e^{-u^{2}} u d u & =\left(-\frac{e^{-u^{2}}}{2}\right)_{c^{1 / 2} \alpha^{\zeta} n^{\zeta-1 / 2}}^{\infty} \\
& =(n / 2 c) e^{-c \alpha^{2 \zeta} n^{2 \zeta-1}}
\end{aligned}
$$

In the rest of this chapter trivial and rather

redundant corollaries of some of the theorems are stated explicitly for ease of later reference.

(2.3) LEMMA.

$$
\begin{aligned}
& \left|\sum_{h=-\infty}^{\infty} e^{-c h^{2} / n}-2 \int_{0}^{\infty} e^{-c t^{2} / n} d t\right| \\
= & \left|\sum_{h=-\infty}^{\infty} e^{-c h^{2} / n}-(\pi n / c)^{2 / 2}\right|<1 .
\end{aligned}
$$

PROOF.

$$
\begin{aligned}
& \text { Let } S=\sum_{h=1}^{\infty} e^{-c h^{2} / n} \text { is lnat } \\
& \sum_{n=-\infty}^{\infty} e^{-\operatorname{ch}^{2} / n}=1+\because \cdots \quad \text { l.t: } \quad r(t)=e^{-c t^{2} / n} .
\end{aligned}
$$


Then since $f^{\prime}(t)=-(2 c t / n) e^{-c t^{2} / n}$, it is easily seen that $f$ takes its maximum value of 1 at $t=0$ and is monotone decreasing (strictly) for $t>0$. Letting

$$
\begin{aligned}
& a(t)=e^{-\operatorname{ch}^{2} / n}, \quad h \leq t<h+1, \\
& b(t)=e^{-c(h+1)^{2} / n}, \quad h \leq t<h+1,
\end{aligned}
$$

we have

$$
b(t)<f(t) \leq a(t), 0 \leq t<\infty
$$

Thus

$$
S=\int_{0}^{\infty} b(t) d t<\int_{0}^{\infty} f(t) d t<\int_{0}^{\infty} a(t) d t=1+S .
$$

Since $\int_{0}^{\infty} f(t) d t=(1 / 2)(\pi n / c)^{I / 2}$ by $(2.1)$, we have

$$
s<(1 / 2)(\pi n / c)^{1 / 2}<s+1
$$

Thus

$$
(\pi n / c)^{1 / 2}-1<2 S+1<(\pi n / c)^{1 / 2}+1 \text {. The theorem }
$$

follows.

The following corollaries are immediate.

(2.4) COROLLARY.

$$
\sqrt{c / \pi n} \sum_{n=-\infty}^{\infty} e^{-c h^{2} / n}=1+0\left(n^{-1 / 2}\right) \text {, uniformzy in }
$$

any finite interval $0 \leq \mathrm{c} \leq \mathrm{K}$. 
Chap. 2

(2.5) COROLLARY.

$$
\sum_{n=-\infty}^{\infty} e^{-c h^{2} / n}=0\left(\int_{0}^{\infty} e^{-c t^{2} / n} d t\right) .
$$

(2.6) LEMMA.

$$
\begin{aligned}
\sum_{h=-\infty}^{\infty}|h| e^{-c h^{2} / n} & \geq 2 \int_{0}^{\infty} e^{-c t^{2} / n} t d t-(2 n / e c)^{1 / 2} \\
& =(n / c)-(2 n / e c)^{1 / 2}
\end{aligned}
$$

For a sufficiently large,

$$
\begin{aligned}
\sum_{h=-\infty}^{\infty}|h| e^{-c h^{2} / n} & \leq 2 \int_{0}^{\infty} e^{-c t^{2} / n} t d t+(2 n / e c)^{1 / 2} \\
& =(n / c)+(2 n / e c)^{1 / 2} .
\end{aligned}
$$

PROOF.

$$
\begin{aligned}
& \text { Let } S=\sum_{h=1}^{\infty} h e^{-c h^{2} / n} \text { so that } \sum_{h=-\infty}^{\infty}|h| e^{-c h^{2} / n}=2 S . \\
& \text { Let } f(t)=t e^{-c t^{2} / n} .
\end{aligned}
$$

Then

$$
f:(t)=e^{-c t^{2} / n}\left(1-\frac{2 c t^{2}}{n}\right)
$$

and

$$
f^{\prime \prime}(t)=\frac{2 c t}{n} e^{-c t^{2} / n}\left(\frac{2 c t^{2}}{n}-3\right) .
$$


Chap. 2

18

It is easily verified that $f$ is monotone increasing for

$0 \leq t \leq(n / 2 c)^{1 / 2}$, monotone decreasing for $t \geq(n / 2 c)^{1 / 2}$,

and takes a maximum value of $(n / 2 e c)^{1 / 2}$ when $t=(n / 2 c)^{1 / 2}$.

Hence $f$ is concave downward for $0 \leq t \leq(3 \mathrm{n} / 2 \mathrm{c})^{1 / 2}$.

Choose an integer $h_{0}$ such that $h_{0}-1<(n / 2 c)^{1 / 2} \leq h_{0}$

Since

$$
\sqrt{3}\left(\frac{n}{2 c}\right)^{1 / 2} \geq\left(\frac{n}{2 c}\right)^{1 / 2}+1>h_{0} \text { if } n>(2+\sqrt{3}) c \text {, }
$$

it follows that $f$ is concave downward for $h_{0}-1 \leq t \leq h_{0}$ when $n>(2+\sqrt{3}) c$.

$$
\begin{array}{ll}
\text { Set } a(t)=h e^{-c h^{2} / n} & , h \leq t<h+1, \\
b(t)=(h+1) e^{-\frac{c(h+1)^{2}}{n}} & , h \leq t<h+1 .
\end{array}
$$

Then

$$
\begin{aligned}
& b(t)<f(t) \leq a(t) \text { for } t \geq h_{0}, \\
& a(t) \leq f(t)<b(t) \text { for } 0 \leq t \leq h_{0}-1 .
\end{aligned}
$$

Now

$$
\begin{aligned}
\int_{0}^{h}(b(t)-a(t)) d t & =\sum_{h=1}^{h} h e^{-c h^{2} / n}-\sum_{h=0}^{h} h e^{-c h^{2} / n} \\
& =h_{0} e^{-c h_{0}^{2} / n}=f\left(h_{0}\right)
\end{aligned}
$$


Thus

$$
\begin{aligned}
& \int_{0}^{\infty} f(t) d t \leq \int_{0}^{h_{0}^{-1}} b(t) d t+\int_{h_{0}^{-1}}^{h_{0}} f(t) d t+\int_{h_{0}}^{\infty} a(t) d t \\
& =\int_{0}^{\infty} a(t) d t+\int_{0}^{h}(b(t)-a(t)) d t+\int_{h_{0}-1}^{h_{0}}(f(t)-b(t)) d t \\
& \leq s+h_{0} e^{-c h_{0}^{2} / n}+\left((n / 2 e c)^{1 / 2}-h_{0} e^{\left.-c h_{0}^{2} / n\right)}\right. \\
& =s+(n / 2 e c)^{1 / 2} .
\end{aligned}
$$

Thus

$$
\begin{aligned}
\sum_{h=-\infty}^{\infty}|h| e^{-c h^{2} / n} & \geq \int_{0}^{\infty} 2 e^{-c t^{2} / n} t d t-(2 n / e c)^{1 / 2} \\
& =(n / c)-(2 n / e c)^{1 / 2} .
\end{aligned}
$$

Also

$$
\begin{aligned}
& \int_{0}^{\infty} f(t) d t \geq \int_{0}^{h} a(t) d t+\int_{h_{0}^{-1}}^{h} f(t) d t+\int_{h_{0}^{-1}}^{q} b(t) d t \\
& =\int_{0}^{\infty} b(t) d t-\int_{0}^{h_{0}}(b(t)-a(t)) d t-\int_{h_{0}^{-1}}^{h_{0}} a(t) d t+\int_{h_{0}^{-I}}^{h_{0}} f(t) d t \\
& =S-f\left(h_{0}\right)-f\left(h_{0}-1\right)+\int_{h_{0}-1}^{h} f(t) d t \\
& =s-\int_{h_{0}^{-1}}^{h_{0}} f(t) d t+2\left(\int_{h_{0}-1}^{h_{0}} f(t) d t-\frac{f\left(h_{0}-1\right)+f\left(h_{0}\right)}{2}\right)
\end{aligned}
$$


Chap. 2

$\geq s-\int_{b_{0}-1}^{h_{0}} f(t) d t$ if $n>(2+\sqrt{3}) c$, since $f$ is then concave downward in $h_{0}-1 \leq t \leq h_{0}$,

$\geq s-(n / 2 e c)^{1 / 2}$

Thus for $n$ sufficiently large (certainly if $n>(2+\sqrt{3}) c$ )

$$
\begin{aligned}
\sum_{n=-\infty}^{\infty}|h| e^{-c h^{2} / n} & \leq 2 \int_{0}^{\infty} e^{-c t^{2} / n} t d t+(2 n / e c)^{1 / 2} \\
& =(n / c)+(2 n / e c)^{1 / 2} .
\end{aligned}
$$

The following corollary is immediate.

(2.7) COROLLARY.

$$
\sum_{h=-\infty}^{\infty}|h| e^{-c h^{2} / n}=0\left(\int_{0}^{\infty} e^{-c t^{2} / n} t d t\right) .
$$

(2.8) LEMMA.

For $1 / 2<\zeta<2 / 3$,

$|h|>\left(\alpha_{n}\right)^{\zeta}|h| e^{-c h^{2} / n}=0\left(\int_{(\alpha n)^{\zeta}}^{\infty} e^{-c t^{2} / n} t d t\right)$.

PROOF.

Since $\zeta>1 / 2,(\alpha n)^{\zeta}>(n / 2 c)^{1 / 2}+1$ for $n$ sufficiently large, and thus, with the notation used in the proof of The orem (2.6)

$$
b(t)<f(t) \text { for } t \geq(\alpha n)^{5} \text {. }
$$


Chep. 2

Then

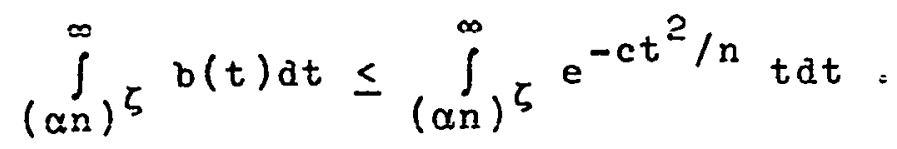

That is

$$
\sum_{h>(\alpha n)^{\zeta}} h e^{-c h^{2} / n} \leq \int_{(\alpha n)^{\zeta}}^{\infty} e^{-c t^{2} / n} t d t .
$$

Therefore

$$
|h|>(\alpha n)^{\zeta} h e^{-c h^{2} / n} \leq 2 \int_{(\alpha n)^{\zeta}}^{\infty} e^{-c t^{2} / n} t d t
$$

and the result follows.

(2.9) LEMMA.

$$
\begin{aligned}
\sum_{n=-\infty}^{\infty}|h|^{3} e^{-c h^{2} / n} & \geq 2\left(\int_{0}^{\infty} e^{-c t^{2} / n} t^{3} d t-(3 n / 2 e c)^{3 / 2}\right) \\
& =\left(n^{2} / c^{2}\right)-\frac{1}{\sqrt{2}}(3 n / e c)^{3 / 2} .
\end{aligned}
$$

For n sufficiently large,

$$
\begin{aligned}
\sum_{n=-\infty}^{\infty}|h|^{3} e^{-c h^{2} / n} & \geq 2\left(\int_{0}^{\infty} e^{-c t^{2} / n} t^{3} d t+(3 n / 2 e c)^{3 / 2}\right) \\
& =\left(n^{2} / c^{2}\right)+\frac{1}{\sqrt{2}}(3 n / e c)^{3 / 2} .
\end{aligned}
$$

PROOF.

$$
\text { Let } S=\sum_{h=1}^{\infty} h^{3} e^{-c h^{2} / n} \text { so that }
$$


Chap. 2

22

$$
\sum_{h=-\infty}^{\infty}|h|^{3} e^{-c h^{2} / n}=25 \text {. Let } g(t)=t^{3} e^{-c t^{2} / n} \text {. }
$$

Then

$$
g^{\prime}(t)=t^{2} e^{-c t^{2} / n}\left(3-\frac{2 c}{n} t^{2}\right)
$$

and

$$
g^{\prime \prime}(t)=2 t e^{-c t^{2} / n}\left(2 \frac{c}{n} t^{2}-1\right)\left(\frac{c}{n} t^{2}-3\right) \text {. }
$$

It is easily verified that $g$ is monotone increasing for $0 \leq t \leq(3 n / 2 c)^{1 / 2}$, monotone decreasing for $t \geq(3 n / 2 c)^{1 / 2}$, and takes a maximum value of $(3 n / 2 e c)^{3 / 2}$ when $t=(3 n / 2 c)^{1 / 2}$. For $(n / 2 c)^{1 / 2} \leq t \leq(3 n / c)^{1 / 2}, g$ is concave downward.

Choose an integer $h_{0}$ such that $h_{0}-1<(3 n / 2 c)^{1 / 2} \leq h_{0}$.

Since

$$
h_{0}-1 \geq \sqrt{3}(n / 2 c)^{1 / 2}-1 \geq(n / 2 c)^{1 / 2} \text { if } n \geq(2+\sqrt{3}) c,
$$

and

$$
\begin{aligned}
& h_{0}<\frac{1}{\sqrt{2}}(3 n / c)^{1 / 2}+1 \leq(3 n / c)^{1 / 2} \\
& \text { if } n \geq\left(2+\frac{4}{3} \sqrt{2}\right) c \quad(\text { which exceeds }(2+\sqrt{3}) c),
\end{aligned}
$$

it follows that $g$ is concave downward for $h_{0}-1 \leq t \leq h_{0}$,

when $n \geq\left(2+\frac{4}{3} \sqrt{2}\right) c$ 


$$
\begin{aligned}
\text { Set } a(\dot{\tau})=n^{3} e^{-c h^{2} / n} & , n \leq t<n+1, \\
b(t)=(h+1)^{3} e^{-c(h+l)^{2} / n} & , h \leq t<h+1 .
\end{aligned}
$$

Now $b(t)<g(t) \leq a(t))$ for $t \geq h_{0}$,

$$
a(t) \leq g(t)<b(t) \text { for } 0 \leq t \leq h_{0}-1 \text {, }
$$

and proceeding just as in the proof of Theorem (2.6) we obtain :

$$
\begin{aligned}
& \int_{0}^{h}(b(t)-a(t)) d t=h_{0} 3 e^{-c h_{0}^{2} / n}=g\left(h_{0}\right) \\
& \int_{0}^{\infty} g(t) d t \leq s+g\left(h_{0}\right)+\left\{(3 n / 2 e c)^{3 / 2}-g\left(h_{0}\right)\right\} \\
&=s+(3 n / 2 e c)^{3 / 2}
\end{aligned}
$$

whence

$$
\begin{aligned}
& \sum_{n=-\infty}^{\infty}|h|^{3} e^{-c h^{2} / n} \geq 2\left(\int_{0}^{\infty} e^{-c t^{2} / n} t^{3} d t-(3 n / 2 e c)^{1 / 2}\right) \\
& =\left(n^{2} / c^{2}\right)-\frac{1}{\sqrt{2}}(3 n / e c)^{3 / 2} . \\
& \int_{0}^{\infty} g(t) d t \geq s-\int_{h_{0}^{-1}}^{h_{0}} g(t) d t+2\left\{\int_{h_{0}^{-1}}^{h_{0}} g(t) d t-\frac{g\left(h_{0}-1\right)+g\left(h_{0}\right)}{2}\right\} \\
& \geq s-(3 n / 2 e c)^{3 / 2} \text { if } n>\left(2+\frac{4}{3} \sqrt{2}\right) c
\end{aligned}
$$

(and therefore if $n>4 c$ ) since $g$ is then concave downward

in $h_{0}-1 \leq t \leq h_{0}$. Thus for $n$ sufficiently large 
Chap. 2

24

$$
\begin{aligned}
& \left(\operatorname{certainz} \mathrm{y} \text { if } \mathrm{a}>\left(2 \div \frac{4}{3} \sqrt{2}\right) \mathrm{c}\right) \text {, } \\
& \sum_{h=-\infty}^{\infty}|h|^{3} e^{-c h^{2} / n} \leq 2\left(\int_{0}^{\infty} e^{-c t^{2} / n} t^{3} d t+(3 n / 2 e c)^{1 / 2}\right) \\
& =\left(n^{2} / c^{2}\right)+\frac{1}{\sqrt{2}}(3 n / e c)^{3 / 2} .
\end{aligned}
$$

The following corollary is immediate.

(2.10) COROLLARY.

$$
\sum_{h=-\infty}^{\infty}|h|^{3} e^{-c h^{2} / n}=0\left(\int_{0}^{\infty} e^{-c t^{2} / n} t^{3} d t\right) .
$$




\section{CHAPTER 3 \\ SUMMAB ILITY $(e, c)$}

In this chapter a method of summation $(e, c)$ is defined and it is shown that if either $a_{n}=o(1)$ or $A_{n}=o\left(n^{1 / 2}\right)$ then summability $(B, \alpha, \beta)$ implies summability $(e, \alpha / 2)$. (In the note at the end of the chapter it is observed that these methods are actuaily equivalent under either condition.)

For $\mathrm{c}>0$, summability $(\mathrm{e}, \mathrm{c})$ is defined by saying

that $\sum_{n=0}^{\infty} a_{n}=A(e, c)$

if $\sqrt{c / \pi n} \sum_{h=-\infty}^{\infty} e^{-c h^{2} / n} A_{n+h}+A$ when $n+\infty$.

(Recall that $A_{m}=0$ for $\left.m<0.\right)$

For this definition and results for Borel summability $B$ anglogous to those which follow for summability $(B, \alpha, \beta)$ see Hardy $[8, \$ 9.10]$ and the references given there. Part (i) of Theorem (3.1) is proved in Borwein [5], while part (ii) is proved (with details omitted) in Hardy [8, Theorem 150]. 
(3.1) THEOREM.

$$
\begin{aligned}
& \text { If } a_{n}=o(I) \text { and } \\
& \text { either (i) } \sum_{n=0}^{\infty} a_{n}=A(B, \alpha, \beta) \\
& \text { or (ii) } \sum_{n=0}^{\infty} a_{n}=A(e, c), \\
& \text { then } A_{n}=o\left(n^{1 / 2}\right) .
\end{aligned}
$$

PROOF.

The result for case (i) is just (1.2) with $\rho=0$. Suppose now that condition (ii) holds.

Since

$$
\sqrt{c i \pi n} \sum_{h=-\infty}^{\infty} e^{-c h^{2} / n}=I+o(I) \text { by }(2.4)
$$

it follows that

$$
\text { (3.2) } \begin{aligned}
\sqrt{c / \pi n} & \sum_{n=-\infty}^{\infty}\left(A_{n+h}-A_{n}\right) e^{-c h^{2} / n} \\
= & A+o(1)-A_{n}\{1+o(1)\} \quad \\
& \text { Since } a_{n}+0, \text { it is well known that } A_{n}=o(n) .
\end{aligned}
$$

Thus

$$
\Lambda_{n+n}=0(n+|n|) \text { by }(1.10) \text { with } \lambda=1 \text {, }
$$


Chap. 3

and therefore

$$
A_{n+n}-A_{n}=O(n+|n|)+O(n)=O(n+|n|) .
$$

Thus

$\sqrt{c / \pi n}|h|>n \zeta\left(A_{n+h}-A_{n}\right) e^{-c h^{2} / n}$

$=\sqrt{c / \pi n} \sum_{|h|>n} O(n+|h|) e^{-c h^{2} / n}$

$=0\left\{n^{-1 / 2}\left[n \sum_{|n|>n} e^{-c h^{2} / n}+\sum_{|h|>n}|h| e^{-c h^{2} / n}\right)\right\}$

$=0\left\{n^{1 / 2} \sum_{|h|>n} \zeta|h| e^{-c h^{2} / n}\right\}$

$=0\left\{n^{1 / 2} \int_{n}^{\infty} e^{-c t^{2} / n} t d t\right\}$

by (2.8) with $\alpha=1$

$=0\left\{n^{1 / 2}\left((-n / 2 c) e^{-c t^{2} / n}\right)_{n}^{\infty}\right\}$

$=0\left\{n^{3 / 2} e^{-c n^{2 \zeta-1}}\right\}$

$=o(1)$ as $n \rightarrow \infty$ since $2 \zeta-1>0$. 
Also

$$
\begin{aligned}
& \sqrt{c / \pi n}|h| \leq n \zeta\left(A_{n+h}-A_{n}\right) e^{-c h^{2} / n} \\
& =\sqrt{c / \pi} n^{-1 / 2} \sum_{|n| \leq n} \circ(|n|) e^{-c^{2} / n} \quad \text { by (I.II) with } j=n \\
& =0\left(n^{-1 / 2} \sum_{|h| \leq n} 5|h| e^{-c h^{2} / n}\right) \\
& \text { since (1.11) holds uniformly for }|h| \leq n^{\zeta} \\
& =0\left(n^{-1 / 2} \int_{0}^{\infty} e^{-c t^{2} / n} t d t\right) \quad \text { by }(2.7) \\
& =0\left(n^{-1 / 2}(n / 2 c) \quad \text { by }(2.1)\right. \\
& =o\left(n^{1 / 2}\right) \text {. }
\end{aligned}
$$

Therefore

$$
\begin{aligned}
& \sqrt{c / \pi n} \sum_{h=-\infty}^{\infty}\left(A_{n+h}-A_{n}\right) e^{-c h^{2} / n}=o(1)+o\left(n^{1 / 2}\right)=o\left(n^{1 / 2}\right) \\
& \text { It follows from }(3.2) \text { that } \\
& A_{n}(1+o(1))=A+o(1)+o\left(n^{1 / 2}\right)=o\left(n^{1 / 2}\right)
\end{aligned}
$$

Therefore $A_{n}=o\left(n^{1 / 2}\right)$. 
Chap. 3

We now prove as lemmas four results which will be needed in the proof of Theorem (3.8). In each of these lemmas we let $h=m-n=m-x / \alpha$, choose $\frac{1}{2}<\zeta<\frac{2}{3}$, and assume the condition

(3.3) $\dot{A}_{n}=o\left(n^{1 / 2}\right)$.

(3.4) LEMMA.

$$
e^{-\alpha n}|h|>(\alpha n)^{5 A_{m}} \frac{(\alpha n)^{\alpha m+\beta-1}}{\Gamma(\alpha m+\beta)}=o(1) \text { as } n+\infty .
$$

PROOF.

$$
\begin{aligned}
& e^{-\alpha n}|h|>(\alpha n)^{5} A_{m} \frac{(\alpha n)^{\alpha m+\beta-1}}{\Gamma(\alpha m+\beta)} \\
& =0\left(e^{-\alpha n}|h|>(\alpha n)^{5^{2}} m^{2 / 2} \frac{(\alpha n)^{\alpha m+\beta-1}}{\Gamma(\alpha m+\beta)}\right) \\
& \text { since certainly } A_{m}=0\left(m^{1 / 2}\right) \text { by }(3.3) \\
& =0\left((\alpha n)^{1 / 2} e^{-\alpha n} \sum_{|h|>(\alpha n)^{5}} \frac{(\alpha n)^{\alpha m+\beta-3 / 2}}{\Gamma(\alpha m+\beta-1 / 2)}\right) \quad \text { by }(1.7) \\
& =0\left[\left(\alpha_{n}\right)^{I / 2} e^{-(\alpha n)^{\eta}}\right) \text { where } 0<n<2 \zeta-1 \\
& \text { by (1.3) with } x=\alpha n \\
& =O(I) \text { as } n \rightarrow \infty \text {. }
\end{aligned}
$$

(3.5) LEMMA.

$$
\mid h \sum_{\leq(\alpha n)^{\zeta}} e^{-\alpha h^{2} / 2 n} A_{n+h} O\left(\frac{\lfloor h \mid+1}{n}\right)=o\left(n^{1 / 2}\right) \text { as } n+\infty \text {. }
$$


PROOF.

$$
\begin{aligned}
& |h| \leq(\alpha n)^{5} e^{-\alpha h^{2} / 2 n} A_{n+h} O\left(\frac{|h|+1}{n}\right) \\
& =\mid h \sum_{\mid \leq(\alpha n)^{5}} e^{-\alpha h^{2} / 2 n} \circ\left(n^{1 / 2}\right) \circ\left(\frac{|h|+1}{n}\right) \text { by (1.12) with } j=n \\
& =o\left(n^{-1 / 2}\right) \mid n \sum_{\mid \leq(\alpha n)^{5}} e^{-\alpha h^{2} / 2 n} o(|h|+1) \\
& \text { since (1.12) holds uniformly for }|h| \leq\left(\alpha_{n}\right)^{\zeta} \\
& =0\left(n^{-1 / 2} \mid h \sum_{\leq(\alpha n)^{5}} e^{-\alpha h^{2} / 2 n}(|h|+1)\right) \\
& =0\left(n^{-1 / 2} \int_{0}^{\infty} e^{-\alpha t^{2} / 2 n}(t+1) d t\right) \quad \text { by (2.5) and (2.7) } \\
& =o\left(n^{-1 / 2}\{(n / \alpha)+(1 / 2) \sqrt{2 \pi n / \alpha}\}\right) \quad \text { by }(2.1) \\
& =o\left(n^{1 / 2}\right)+o(1) \\
& =o\left(n^{1 / 2}\right)
\end{aligned}
$$

(3.6) LEMMA.

$$
|h| \leq(\alpha n)^{5} e^{-\alpha h^{2} / 2 n} A_{n+h} 0\left(\frac{|h|^{3}}{n^{2}}\right)=o\left(n^{1 / 2}\right) \text { as } n \rightarrow \infty \text {. }
$$


PROOF.

$$
\begin{aligned}
& |h| \leq(\alpha n)^{5} e^{-\alpha h^{2} / 2 n} A_{n+h} \circ\left(\frac{|n|^{3}}{n^{2}}\right) \\
& =\sum_{|h| \leq(\alpha n)^{\zeta}} e^{-\alpha h^{2} / 2 n} \circ\left(n^{1 / 2}\right) \circ\left(\frac{|n|^{3}}{n^{2}}\right) \quad \text { by (1.12) with } j=n \text {. } \\
& =o\left\{n^{-3 / 2}|h|_{\mid \leq(\alpha n)^{5}} e^{-\alpha h^{2} / 2 n}|h|^{3}\right\} \\
& \text { since (1.12) holds uniformly for }|h| \leq(\alpha n)^{\zeta} \\
& =o\left\{n^{-3 / 2} \int_{0}^{\infty} e^{-\alpha t^{2} / 2 n} t^{3} d t\right\} \quad \text { by }(2.10) \\
& =\circ\left\{n^{-3 / 2}\left(2 n^{2} / \alpha^{2}\right)\right\} \quad \text { by }(2.1) \\
& =o\left(n^{1 / 2}\right) \text {. }
\end{aligned}
$$

(3.7) LEMMA.

$$
|h|>(\alpha n)^{\zeta} e^{-\alpha h^{2} / 2 n} A_{n+h}=o(I) \quad \text { as } n+\infty \text {. }
$$

PROOF.

$$
\begin{aligned}
& |h|>(\alpha n)^{\zeta} e^{-\alpha h^{2} / 2 n} A_{n+h} \\
= & |h|>(\alpha n)^{\zeta} e^{-\alpha h^{2} / 2 n} o(|h|) \quad \text { by }(1.13)
\end{aligned}
$$




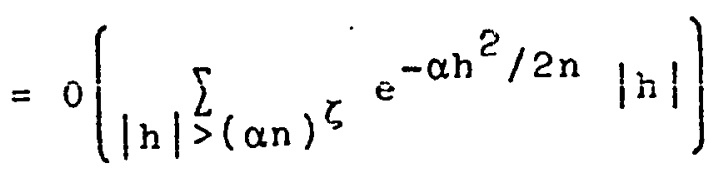

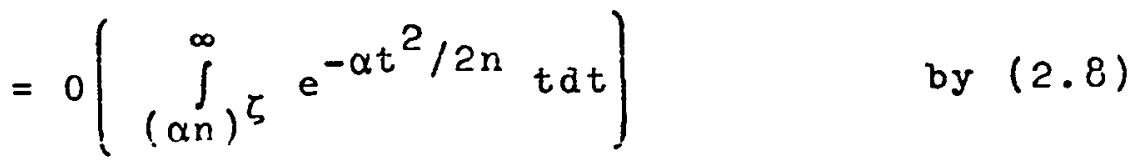

$=0\left[(n / \alpha) e^{-\alpha^{2 \zeta+1} n^{2 \zeta-1} / 2}\right] \quad$ by $(2.2)$

$=0\left(n e^{-\rho n^{a}}\right) \quad$ where $\rho=\frac{\alpha}{2}^{2 \zeta+1}>0$

$$
\text { and } a=2 \zeta-1>0
$$

$=o(1)$ as $\mathrm{n} \rightarrow \infty$.

(3.8) THEOREM.

$$
\text { If } A_{n}=o\left(n^{1 / 2}\right) \text {, then } \sum_{n=0}^{\infty} a_{n}=A(B, \alpha, \beta)
$$

if and onity if $\sum_{n=0}^{\infty} a_{n}=A(e, \alpha / 2)$.

PROOF.

We first observe that if the theorem holds for $A=0$

it holds for arbitrary $A$.

For since

$\alpha e^{-x} \sum_{m=i n}^{\infty} \frac{y^{(x m+\beta-1}}{\Gamma(\alpha m+\beta)}+1$ as $x \rightarrow \infty$

by the regulanity of the $(1, \alpha, \beta)$ method of 
summation, we have

(3.9) $\alpha e^{-x} \sum_{m=N}^{\infty}\left(A_{m}-A\right) \frac{x^{\alpha m+\beta-i}}{\Gamma(\alpha m+\beta)}+0$ as $x \rightarrow \infty$ if and only if (3.10) $\alpha e^{-x} \sum_{m=N}^{\infty} A_{m} \frac{x^{\alpha i m+\beta-1}}{\Gamma(\alpha m+\beta)}+A$ as $x+\infty$.

Also since

$$
\sqrt{\alpha / 2 \pi n} \sum_{h=-\infty}^{\infty} e^{-\alpha h^{2} / 2 n}+1 \text { as } n \rightarrow \infty \text { by }(2.4)
$$

$(3.11) \cdot \sqrt{\alpha / 2 \pi n} \sum_{h=-\infty}^{\infty} e^{-\alpha h^{2} / 2 n}\left(A_{n+h}-A\right)+0$ as $n \rightarrow \infty$ if and only if

(3.12) $\sqrt{\alpha / 2 \pi n} \sum_{h=-\infty}^{\infty} e^{-\alpha h^{2} / 2 n} A_{n+h}+A$ as $n+\infty$.

Thus assuming the result for limit 0 , we have

$$
(3.10) \Longleftrightarrow(3.9) \Longleftrightarrow(3.11) \Longleftrightarrow(3.12) \text { and the }
$$

result for limit A follows.

We therefore assume as we may that $A=0$. We

further assume, without loss in generality, that

$$
a_{0}=\ldots=a_{N-1}=0 \text {, so that } A_{m}=0 \text { if } m<N \text {. }
$$

Now let $x=\alpha r_{1}, \frac{1}{2}<\zeta<\frac{2}{3}$, and $h=m-n=m-x / \alpha$.

Then

$$
e^{-\alpha n} \sum_{m=1}^{\infty} A_{m} \frac{(\alpha n)^{\alpha m+\beta-1}}{\Gamma(\alpha m+\beta)}
$$




$$
\begin{aligned}
& =\mathrm{e}^{-\alpha \mathrm{n}} \sum_{|\mathrm{h}|^{>}(\alpha \mathrm{n})^{\zeta \mathrm{A}_{\mathrm{m}}} \frac{(\alpha \mathrm{n})^{\alpha m+\beta-1}}{\Gamma(\alpha m+\beta)}} \\
& +\mathrm{e}^{-\alpha n} \sum_{|\mathrm{n}| \leq(\alpha \mathrm{n})} \mathrm{A}_{\mathrm{m}} \frac{(\alpha \mathrm{n})^{\alpha \mathrm{m}+\beta-1}}{\Gamma(\alpha \mathrm{m}+\beta)} \\
& =o(1)+n^{-1 / 2}\left\{\sum_{|h| \leq(\alpha n)} \zeta^{e^{-\alpha h^{2} / 2 n} A_{n+h}}\right. \\
& +\mid h \sum_{\leq(\alpha n)} s^{e^{-\alpha h^{2} / 2 n} A_{n+h}} 0\left(\frac{|h|+l}{n}\right)
\end{aligned}
$$

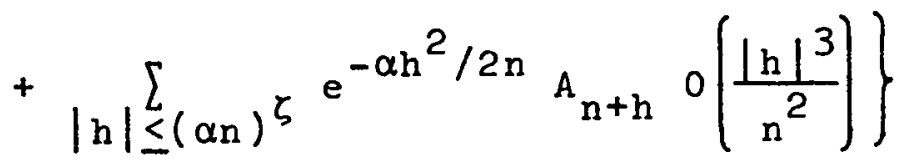

$$
\begin{aligned}
& \text { by }(3.4) \text { and }(1.5) \text { with } x=\alpha_{n} \\
& =o(1)+n^{-1 / 2} \sum_{|n| \leq(\alpha n)^{\zeta}} e^{-\alpha h^{2} / 2 n} A_{n+h}+o(I)+o(I) \\
& \text { by }(3.5) \text { and }(3.6) \\
& =n^{-1 / 2}|n| \leq(\alpha n)^{5} e^{-\alpha h^{2} / 2 n} A_{n+h}+o(1) .
\end{aligned}
$$

Since

$$
|h|>(\alpha n) \sum^{e^{-\alpha h^{2} / 2 n} A_{n+h}=o(1)} \quad \text { by }(3.7)
$$

it follows that

(3.13) $e^{-\alpha n} \sum_{m=N}^{\infty} \Lambda_{m} \frac{(\alpha n)^{\alpha m+\beta-1}}{\Gamma(\alpha i n+\beta)}=o(1)$ as $n \rightarrow \infty$ 
if and only if

$$
n^{-1 / 2} \sum_{n=-\infty}^{\infty} e^{-\alpha h^{2} / 2 n} A_{n+h}=o(1) \quad \text { as } n+\infty
$$

i.e. if and only if $\sum_{n=0}^{\infty} a_{n}=0(e, \alpha / 2)$.

\section{Since}

$$
\sum_{n=0}^{\infty} a_{n}=0(B, \alpha, \beta)
$$

implies

$$
e^{-\alpha n} \sum_{m=N}^{\infty} A_{m} \frac{(\alpha n)^{\alpha m+\beta-1}}{\Gamma(\alpha m+\beta)}=o(1) \text { as } n \rightarrow \infty
$$

the theorem is established ir one direction. (The rast of the other direction is established in the note at the end of this chapter.)

(3.14) COROLLARY.

$$
\text { If } a_{n}=o(1) \text {, then } \sum_{n=0}^{\infty} a_{n}=A(B, \alpha, \beta)
$$

if and only if $\sum_{n=0}^{\infty} a_{n}=A(e, \alpha / 2)$

PROOF.

The resilt follon: a : vitly from phoorems (3.1) and $(3.8)$. 
NOTE.

Since it is not needed in the proof of the "o" Tauberian Theorem, a proof of the "if" direction of Theorem (3.8) (i.e. that $A_{n}=o\left(n^{1 / 2}\right)$ and summability $(e, \alpha / 2)$ imply summability $(B, \alpha, \beta))$ has been omitted from the body of the thesis. A proof is included here for the sake of completeness and because of the interest of the result.

After (3.13), it is plainly sufficient to show that, if $e^{-x} \sum_{m=N}^{\infty} A_{m} \frac{x^{\alpha m+\beta-1}}{\Gamma(\alpha m+\beta)} \rightarrow 0$ as $x \rightarrow \infty$ through integer multiples of $\alpha$, then it approaches zero as $x \rightarrow \infty$ (without restriction). It is convenient to assume that (1.3) and (1.5) hold with $h=n-[x / \alpha]$ rather than $h=n-x / \alpha$. Conly trivial modifications of Borwein's proof in [5] are needed for this change in h.)

Letting $h=m-n=m-[x / \alpha]$, it follows just as in Lemma (3.4) that

$$
e^{-x} \sum_{n \mid>x} \Lambda_{m} \frac{x^{\alpha m+\beta-1}}{\Gamma(\alpha m+\beta)}=o(1) \text { as } x+\infty \text {. }
$$

NIso, just as in Lemmis $(3.5)$ and $(3.6)$ (with an 
Chap. 3

37

appropriate modification in Lemma (1.12) ), we obtain:

(3.16) $|h| \leq x^{5} e^{-\alpha^{2} h^{2} / 2 x} A_{n+h} 0\left(\frac{|h|+1}{x}\right)=0\left(x^{1 / 2}\right)$ as $x \rightarrow \infty$,

and

$$
\text { (3.17) } \quad \ln \sum_{\leq x^{5}} e^{-\alpha^{2} h^{2} / 2 x} A_{n+h} 0\left(\frac{|h|^{3}}{x^{2}}\right)=0\left(x^{1 / 2}\right) \quad \text { as } x \rightarrow \infty \text {. }
$$

Thus, using $(3.15),(1.5),(3.16)$, and $(3.17)$, we obtain:

(3.18) $e^{-x} \cdot \sum_{m=N}^{\infty} A_{m} \frac{x^{\alpha m+\beta-1}}{\Gamma(\alpha m+\beta)}=\frac{1}{\sqrt{2 \pi x}}|h|_{\leq x} \zeta_{n+n} e^{-\alpha^{2} h^{2} / 2 x}+o(1)$.

Letting $x=\alpha n+k$ where $0 \leq k<\alpha$, it follows

from (3.18) using Lemmas $(3.4),(3.5),(3.6)$ and $(3.7)$,

that it is sufficient to prove that:

$$
(\alpha n)^{-1 / 2} \sum_{|h| \leq x^{5}} e^{-\alpha h^{2} / 2 n} A_{n+h}=o(1) \text { as } n+\infty
$$

implies

$$
\begin{aligned}
& x^{-1 / 2} \sum_{|n| \leq x} e^{-\alpha^{2} h^{2} / 2 x} A_{n+h}=o(1) \text { as } x \rightarrow \infty \\
& \text { Since } x^{-1 / 2}=(\alpha n)^{-1 / 2}+o\left(n^{-3 / 2}\right)
\end{aligned}
$$


chap. 3

and

$$
e^{-\frac{\alpha^{2} n^{2}}{2(\alpha n+k)}}=e^{-\alpha h^{2} / 2 n}\left\{1+0\left[\frac{h^{2}}{n^{2}}\right]\right\}=e^{-\alpha h^{2} / 2 n}\left\{1+0\left(\frac{|h|+1}{n}\right)\right\},
$$

(See the proof of similar results after (4.14))

the result follows. 


\section{CHAPTER 4 \\ SUMMABILITY $(r, k)$}

Our purpose in this chapter is to prove that if

$A_{n}=o\left(n^{1 / 2}\right)$ and $\sum_{n=0}^{\infty} a_{n}=A(e, c)$ then for $0<d<c$,

$\sum_{n=0}^{\infty} a_{n}=A(e, d)($ Theorem 4.23). We do this by first defining

a method of summation $(\gamma, k)$, establishing some properties of it, and eventually linking summability $(\gamma, k)$ to summability

$(e, c)$ where

$c=\frac{k}{2(1-k)}($ Theorem 4.18$)$

For tre definition and preliminary discussion of summability $(\gamma, k)$ see Hardy $[8, \$ 9.11]$. Results $(4.3),(4.4)$, and (4.24) are stated and proved there, while (4.22) is stated with a proof indicated. Results $(4.15),(4.17),(4.18)$, and (4.23) while not stated explicitly are implicit in the material of section 9.11 cited above.

Let $0 \cdot k<1$. Suppose $\sum_{n=0}^{\infty} a_{n} x^{n}$ is convergent for $|x|<1$ and let $f(x)=\sum_{n=0}^{\infty} a_{n} x^{n}$. The Taylor series expansion ${ }^{n}$ or about $x_{0}=1-k$ as centre, valid at least in a disk of radius $k$ is 
Chap. is

40

$$
\begin{aligned}
& \sum_{n=0}^{\infty} \frac{f^{(n)}\left(x_{0}\right)}{n !}\left(x-x_{0}\right)^{n}=\sum_{n=0}^{\infty} \frac{f^{(n)}(1-k)}{n !}(k y)^{n} \\
& =\sum_{n=0}^{\infty} b_{n} y^{n} \text { where } x=1-k+k y \text { and } b_{n}=\frac{f^{(n)}(1-k)}{n !} k^{n} .
\end{aligned}
$$

summability $(\gamma, \mathrm{k})$ is defined by saying that

$$
\begin{aligned}
& \sum_{n=0}^{\infty} a_{n}=A(\gamma, k) \text { if } \sum_{n=0}^{\infty} a_{n} x^{n} \text { converges for }|x|<1 \\
& \text { and } \sum_{n=0}^{\infty} b_{n}=A . \\
& \text { Let } B_{m}=b_{0}+b_{1}+\ldots+b_{m} \text {. Since } \\
& x=1-k+k y \quad, \quad 1-k=k(1-y) \text { and } \frac{1}{1-y}=\frac{k}{1-x}
\end{aligned}
$$

Thus

$$
\begin{aligned}
\sum_{m=0}^{\infty} B_{m} y^{m} & =\frac{I}{1-y} \sum_{m=0}^{\infty} b_{m} y^{m}=\frac{k}{1-x} f(x)=\frac{k}{1-x} \sum_{n=0}^{\infty} a_{n} x^{n} \\
& =k \sum_{n=0}^{\infty} A_{n} x^{n}=k \sum_{n=0}^{\infty} A_{n}(1-k+k y)^{n} .
\end{aligned}
$$

ir ow $k((l-k)+k y)^{n}=\sum_{r=0}^{n}\left(\begin{array}{l}n \\ r\end{array}\right)(I-k)^{n-r} k^{r+l} y^{r}$.

Here $\mathrm{y}^{\mathrm{m}}$ appears only if $\mathrm{n} \geq \mathrm{m}$ and then the coefficient

of $y^{m}$ is $\left(\begin{array}{l}n \\ m\end{array}\right)(1-k)^{n-m} k^{m+1}$.

4.1) Therefore $B_{m}=\sum_{n=m}^{\infty} A_{n}\left(\begin{array}{l}n \\ m\end{array}\right)(1-k)^{n-m} k^{m+1}$

$$
=k^{m+1} \sum_{n=0}^{\infty} A_{m+n}\left(\begin{array}{c}
m+n \\
m
\end{array}\right)(1-k)^{n} .
$$


Chap. 4

Similarly, since

$\sum_{m=0}^{\infty} b_{m^{y}} y^{m}=\sum_{n=0}^{\infty} a_{n} x^{n}=\sum_{n=0}^{\infty} a_{n}(1-k+k y)^{n}$,

. 2) $b_{m}=k^{m} \sum_{n=m}^{\infty} a_{n}\left(\begin{array}{l}n \\ m\end{array}\right)(1-k)^{n-m}$

$$
=k^{m} \sum_{n=0}^{\infty} a_{m+n}\left(\begin{array}{c}
m+n \\
m
\end{array}\right)(1-k)^{n}
$$

.3) THEOREM.

The $(\gamma, k)$ method of summation is regular. PROOF.

It. follows from (4.1) that

$B_{m}=\sum_{n=0}^{\infty} c_{m, n} A_{n}$ where

$c_{m, n}= \begin{cases}0 & , \quad n<m \\ k^{m+1}\left(\begin{array}{l}n \\ m\end{array}\right)(1-k)^{n-m} & , \quad n \geq m . \text { Thus }\end{cases}$

$\sum_{n=0}^{\infty}\left|c_{m, n}\right|=\sum_{n=m}^{\infty} c_{m, n}=k^{m+1} \sum_{n=m}^{\infty}\left(\begin{array}{l}n \\ m\end{array}\right)(1-k)^{n-m}$

$=k^{m+1} \sum_{n=0}^{\infty}\left(\begin{array}{c}m+n \\ m\end{array}\right)(I-k)^{n}$

$=k^{m+1}(1-(1-k))^{-(m+1)}=1$.

$\lim _{m \rightarrow \infty} c_{\tilde{H}, n}=\lim _{m \rightarrow \infty} 0=0$ for all $n$.

The theorem follows from a well known result (sec Hardy [8, 'theorem 2]). 
Note that $(4.1)$ and $(4.2)$ were obtained using the fact that the radius of convergence $R$ of

$\sum_{n=0}^{\infty} a_{n} x^{n}$ is 1 (actually $\geq 1$ but if $R>1, \sum_{n=0}^{\infty} a_{n}$ converges and the method is of no interest).

While a method of summability could be defined by requiring that $B_{m}$ as given by (4.1) exist for all $m$ and tend to $A$ as $m$ tends to $\infty$, it is convenient for our purpose to keep the restriction and some of the following theorems depend upon it.

(4.4) THEOREM.

$$
\text { If } \sum_{n=0}^{\infty} a_{n}=A(y, k) \text { and } 0<\ell<k \text { then }
$$

$\sum_{n=0}^{\infty} a_{n}=A(\gamma, \ell)$.

PROOF.

Let $x=1-k+k y=1-\ell+\ell z$. Then

$\mathrm{ky}=\mathrm{k}-\ell+\ell \mathrm{z}$ and $\mathrm{y}=1-\frac{\ell}{\mathrm{k}}+\frac{\ell}{\mathrm{k}} \mathrm{z}$ where $0<\frac{\ell}{\mathrm{k}}<1$.

Write $f(x)=\sum_{n=0}^{\infty} a_{n} x^{n}=\sum_{n=0}^{\infty} b_{n} y^{n}=\sum_{n=0}^{\infty} c_{n} z^{n}$.

If $\sum_{n=0}^{\infty} a_{n}=A(\gamma, k)$ then $\sum_{n=0}^{\infty} b_{n}=\Lambda$

(by the definition of summability $(\gamma, k)$ ).

Since the $\left(\gamma, \frac{\ell}{k}\right)$ method is recular $\sum_{n=0}^{\infty} b_{n}=A\left(\gamma, \frac{l}{k}\right)$. 
That is $\sum_{n=0}^{\infty} c_{n}=A$. But this is fust summability $(\gamma, l)$.

Therefore $\sum_{n=0}^{\infty} a_{n}=A(\gamma, \ell)$.

The following theorem gives estimates for summability $(\gamma, k)$ similar to those given in Chapter 1 for summability $(B, \alpha, \beta)$. It is essentially Theorem 139 of Hardy [8].

While a proof is outlined there, a much more detailed proof will be given here, because of the interest of the techniques and their similarity to those required to establish the results in Chapter 1 , as well as for the sake of completeness. (4.5) THEOREM.

Suppose that $0<\mathrm{k}<1$ and

$u_{m}=u_{m}(n)=k^{n+1}\left(\begin{array}{l}m \\ n\end{array}\right)(1-k)^{m-n}$ for $m \geq n$

$$
\left(u_{m}=0 \text { for } m<n\right)
$$

so that

$$
\text { (4.6) } \begin{aligned}
\sum_{m=0}^{\infty} u_{m} & =\sum_{m=n}^{\infty} u_{m}=k^{n+1} \sum_{r=0}^{\infty}\left(\begin{array}{c}
n+r \\
n
\end{array}\right)(1-k)^{r} \\
& =k^{n+1}(1-(1-k))^{-(n+1)}=1 .
\end{aligned}
$$

Then

$$
\text { (1) the largest } u_{m} \text { is } u_{M} \text { where } M=[n / k] \text {, }
$$

two terms $u_{n-1}$ and $u_{n}$ being equal if $n / k$ is an integers 
Chep. 4

(2) if $\mathrm{m}=\mathrm{M}+\mathrm{h}$ and $0<\delta<1$, then

$|h|>\delta n=0\left(e^{-\mu n}\right)$ for some positive $\mu=\mu(k, \delta)$;

(3) if $1 / 2<\zeta<2 / 3$ then $\sum_{|\mathrm{h}|>\mathrm{n}} \zeta^{u_{m}}=0\left(\mathrm{e}^{-\mathrm{n}^{n}}\right)$ where $0<n<2 \zeta-1$;

(4) if $|\mathrm{h}| \leq \mathrm{n}^{5}$ and $\mathrm{c}=\frac{\mathrm{k}}{2(1-\mathrm{k})}$ then

(4.7) $u_{m}=\sqrt{c / \pi M} e^{-c h^{2} / M}\left\{1+0\left(\frac{|h|+1}{n}\right)+0\left(\frac{|h|^{3}}{n^{2}}\right)\right\}$

$=\sqrt{c / \pi M} e^{-\operatorname{ch}^{2} / M}\left\{1+0\left(\frac{|h|+1}{M}\right)+0\left(\frac{|h|^{3}}{M^{2}}\right)\right\}$

(5) if $\lambda$ is any real constant

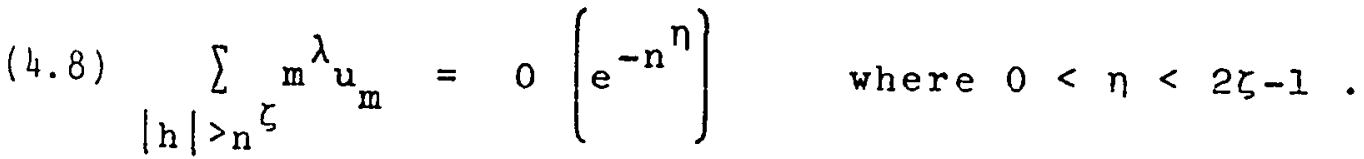

PROOF.

(4.9) $\frac{u_{m}}{u_{m-I}}=\frac{\left(\begin{array}{c}m \\ n\end{array}\right)(1-k)^{m-n}}{\left(\begin{array}{c}m-I \\ n\end{array}\right)(1-k)^{m-n-1}}=\frac{m}{m-n} \quad(1-k)$.

If $m<\frac{n}{k}, k m<n, m-k m>m-n$ and therefore $\frac{m}{m-n}(1-k)>1$.

Thereîore $u_{m}>u_{m-1}$. 
Chap. 4

45

If $m>\frac{n}{k}, k m>n, m-k m<m-n$ and thus $\frac{m}{m-n}(1-k)<1$.

Therefore $u_{m}<u_{m-I}$.

If $m=\frac{n}{k}(=M)$ then $\frac{m}{m-n}(1-k)=1$ and $u_{M}=u_{M-1}$.

This proves part (1).

Now choose $n$ sufficiently large that $n^{\zeta}<\delta n$ and

$n-M<-\delta n$. (We may suppose $0<\delta<\frac{1}{k}-1$ since decreasing

$\delta$ increases the strength of (2). Then if $n$ is sufficiently

large $\delta<\frac{1}{k}-1-\frac{1}{n}$.

Therefore $n+l<\frac{n}{k}-\delta n$,

$$
n-\left(\frac{n}{k}-1\right)<-\delta n,
$$

and $n-M<-\delta n$.

Then $S_{1}$ in what follows does not disappear.)

$$
\begin{aligned}
& \sum_{h=-\infty}^{\infty} u_{M+h} \\
& =\sum_{-M \leq h<-\delta n} u_{M+h}+\sum_{-\delta n \leq h<-n} \zeta^{u_{M+h}}+\mid h \sum_{\leq n} \zeta u_{M+h} \\
& +n^{\zeta}<h \leq \delta n u_{M+h}+\sum_{h>\delta n} u_{M+h}
\end{aligned}
$$


$=\sum_{m=0}^{M_{1}^{-1}} u_{m}+\sum_{m=M_{1}}^{M_{2}-1} u_{m}+\sum_{m=M_{2}}^{M_{3}} u_{m}$

$$
+\sum_{m=M_{3}+1}^{M_{4}} u_{m}+\sum_{m=M_{4}+1}^{\infty} u_{m}
$$

$=s_{1}+s_{2}+s_{3}+s_{4}+s_{5}$

where $M_{1}=M-[\delta n], M_{2}=M-\left[n^{5}\right], M_{3}=M+\left[n^{5}\right]$, and $M_{4}=M+[\delta n]$.

Then

$\mathrm{S}_{1}^{\prime}=\sum_{\mathrm{m}=0}^{\mathrm{M}_{1}-1} \mathrm{~m}^{\lambda} \mathrm{u}_{\mathrm{m}} \leq \sum_{\mathrm{m}=0}^{\mathrm{M}_{1}-1} \mathrm{M}_{1}^{\lambda} \mathrm{u}_{\mathrm{M}_{1}} \leq \mathrm{M}_{1}^{\lambda+1} \mathrm{u}_{\mathrm{M}_{1}} \leq \mathrm{M}^{\lambda+1} \mathrm{u}_{\mathrm{M}_{1}}$. Therefore $S_{1}{ }^{\prime}=0\left(\begin{array}{ll}n^{\lambda+1} & u_{M_{1}} \\ & \end{array}\right)$.

$S_{2}^{\prime}=\sum_{m=M_{1}}^{M_{2}^{-1}} m^{\lambda} u_{m} \leq \sum_{m=M_{1}}^{M_{2}} M_{2}^{\lambda} u_{M_{2}} \leq M_{2}^{\lambda+1} u_{M_{2}} \leq M^{\lambda+1} u_{M_{2}}$.

Therefore $S_{2}{ }^{\prime}=0\left(n^{\lambda+1} u_{M_{2}}\right)$

$s_{4^{\prime}}^{\prime}=\sum_{m=M_{3}+1}^{M_{4}} m^{\lambda} u_{m} \leq \sum_{m=M_{3}+1}^{M_{4}} M_{4}^{\lambda} u_{M_{3}} \leq M_{4}^{\lambda+1} u_{M_{3}}$.

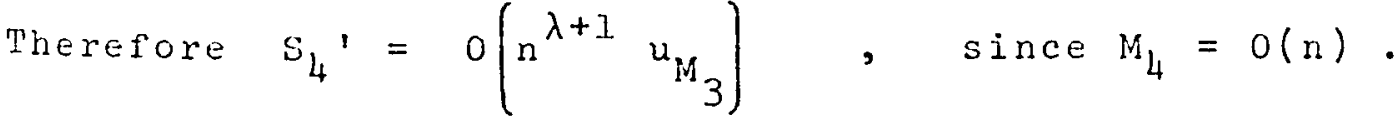


Chap. is

47

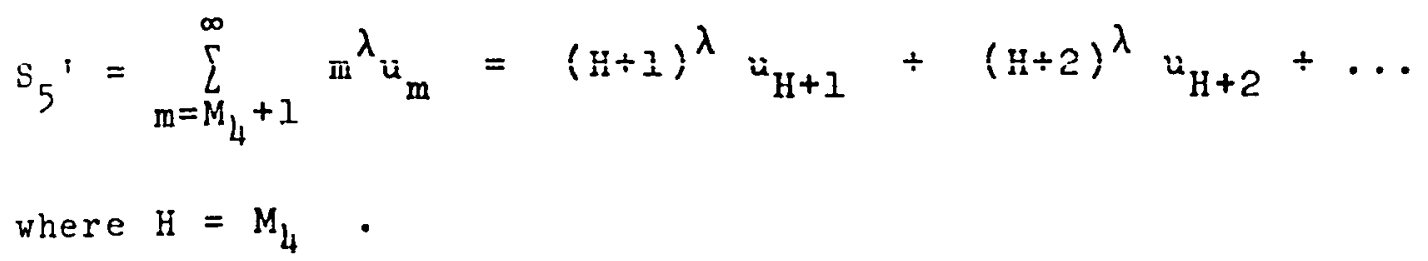

$$
\text { Since } H+2>\frac{n}{k}+\delta n \text {, if } m>H+I \text { then } m>\frac{n}{k}(I+\delta k) \text {. }
$$

Therefore $-n(I+\delta k)>-m k$

and $\quad(1+\delta k) m-(1+\delta k) n>(1+\delta k) m-m k$;

ie. $(1+\delta k)(m-n)>m(1-k+\delta k)$.

Therefore $\frac{m}{m-n}<\frac{1+\delta k}{1-k+\delta k}$.

Thus by $(4.9)$

$$
\begin{aligned}
& \frac{u_{m}}{u_{m-1}}=\frac{m}{m-n}(1-k)<\frac{(1+\delta k)(1-k)}{1-k+\delta k} \\
& =1-\frac{k^{2} \delta}{1-k+\delta k}=1-\delta_{1}=r_{1} .
\end{aligned}
$$

Thus if $m>\mathrm{H}+\mathrm{I}, \frac{\mathrm{u}_{\mathrm{m}}}{\mathrm{u}_{\mathrm{m}-1}}<\mathrm{r}_{1}$ where

$$
1-k<r_{1}<1 \text { since } \delta_{1}<\frac{k^{2} \delta}{k \delta}=k \text { and } \delta_{1}>0 \text {. }
$$

Also since $(H+r)^{\lambda}=r \delta\left(1+\frac{H}{r}\right)^{\lambda} \leq r^{\lambda} 2^{\lambda}$ if $r \geq H$,

while $(H+r)^{\lambda} \leq(2 H)^{\lambda}$ if $r \leq H$,

we have $(H+r)^{\lambda} \leq(2 H)^{\lambda} r^{\lambda}$.

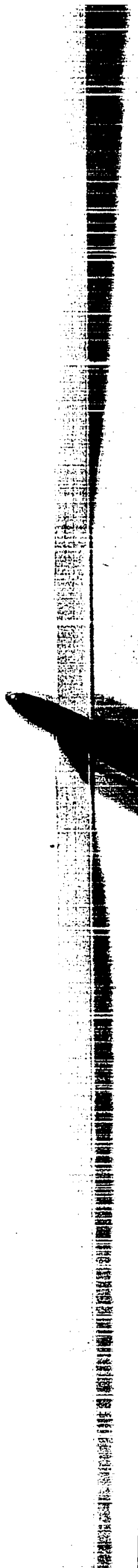


Therefore

$$
\begin{aligned}
& \mathrm{S}_{5}{ }^{\prime} \leq(2 \mathrm{H})^{\lambda} u_{\mathrm{H}+1}\left\{I^{\lambda}+2^{\lambda} \mathrm{r}_{1}+3^{\lambda} \mathrm{r}_{I}{ }^{2}+4^{\lambda} \mathrm{r}_{1}{ }^{3}+\ldots\right\} \\
& \leq(2 \mathrm{II})^{\lambda} \ddot{\leftrightarrow}_{\mathrm{H}}\left(\sum_{n=1}^{\infty} n^{\lambda} r_{I}^{n-1}\right) \\
& =0\left(H^{\lambda} u_{H}\right) \quad \text { since the series converges } \\
& =0\left(\begin{array}{ll}
M_{4}^{\lambda} & u_{M_{4}}
\end{array}\right) \\
& =0\left(\begin{array}{ll}
n^{\lambda} & \\
& u_{4}
\end{array}\right) \text {. }
\end{aligned}
$$

Taking $\lambda=0$ in the $s_{i}^{\prime}, i=1,2,4,5$, gives

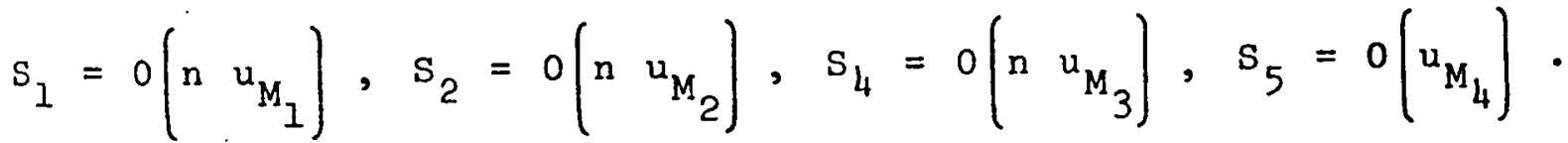

Since $n^{\lambda} e^{-\mu n}=0\left(e^{-\mu_{1} n}\right)$ if $0<\mu_{1}<\mu$,

it therefore suffices to prove that $u_{M_{1}}$ and $u_{M_{4}}$ are $0\left(e^{-\mu n}\right)$ to establish part (2)

$$
\text { Since } e^{-\mu n}=0\left(e^{-n^{n}}\right) \text { it sufrices to prove that }
$$

that $u_{M_{2}}$ anà $u_{M_{3}}$ are $0\left(e^{-n^{n}}\right)$ to establish parts $\operatorname{and}(5)$. 


$$
\text { Now } \ell^{n+1} \sum_{m=n}^{\infty}\left(\begin{array}{l}
m \\
n
\end{array}\right)(1-l)^{m-n}=1 \text { for all } \ell
$$

with $0<\ell<1$.

Therefore $0<\ell^{n+1}\left(\begin{array}{l}m \\ n\end{array}\right)(1-l)^{m-n}<1$.

Thus

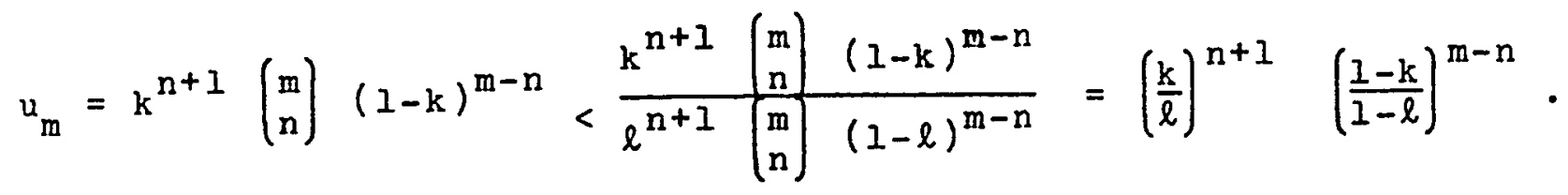

For either $\mathrm{m}=\mathrm{M}_{1}$ or $\mathrm{M}_{4} ; i . e$. for $\mathrm{m}=\mathrm{M} \mp[\delta \mathrm{n}]$,

$$
\begin{aligned}
\left(\frac{k}{l}\right)^{n+1} & \left(\frac{1-k}{1-l}\right)^{m-n} \\
= & \frac{k}{l} \cdot\left(\frac{1-k}{1-l}\right)^{M-(n / k) \pm\{\delta n-[\delta n]\}}\left\{\left(\frac{k}{l}\right)^{n}\left(\frac{1-k}{1-l}\right)^{n / k)-n \mp \delta n}\right\} \\
= & \frac{k}{l}\left(\frac{1-k}{1-l}\right)^{a} \theta^{n} \quad \text { where }-2<a<1 \\
= & 0\left(\theta^{n}\right) \quad \text { where } \theta=\theta(l)=\frac{k}{l}\left(\frac{1-k}{1-l}\right)^{(I / k)-I \mp \delta}
\end{aligned}
$$

$\theta(\ell)=I$ when $\ell=k \cdot \theta(\ell)++\infty$ as $\ell+0$ and as $\ell \uparrow 1$.

$\theta^{\prime}(l)=(1-k)^{(1 / k)-1 \mp \delta} \frac{\ell(1 \mp \delta k)-k}{l^{2}(1-\ell)^{(1 / k+\delta}}$.

Therefore $O^{\prime}(l)=0$ when $\ell=\frac{k}{1 \bar{\mp} \delta k}$.

Thus $O(l)$ has a single minimum when $2=\frac{k}{I \mp \delta k}$. 
Chap. 4

$\theta^{\prime}(k)=\frac{\mp \delta}{1-k} \neq 0$. Hence we can choose $\ell$ such that

$\theta(\ell)<1 . \quad$ (For $m=M_{1}, \theta^{\prime}(k)=\frac{-\delta}{1-k}<0$ so that

we can choose $\ell>k$ such that $\theta(l)<1$. For $m=M_{4}$

$\theta^{\prime}(k)=\frac{\hat{0}}{1-k}>0$ so we can choose $\ell<k$ such that $\theta(\ell)<1$.)

Therefore $u_{M_{1}}$ and $u_{M_{4}}$ are $0\left(\theta_{0}{ }^{n}\right)$ where $0<\theta_{0}<1$.

Let $\mu=\log \frac{1}{\theta_{0}}$. Then $\mu>0$ and $\theta_{0}^{n}=e^{n \log \theta_{0}}=e^{-\mu n}$. Thus $u_{M_{1}}$ and $u_{M_{4}}$ are $O\left(e^{-\mu n}\right)$ where $\mu>0$ and part (2) is established.

We next prove part (4). Suppose $|h| \leq n^{\zeta}$ and write $M=[n / k]=n / k-f$ where $0 \leq f<1$.

Then $\mathrm{m}=\mathrm{M}+\mathrm{h}=\mathrm{n} / \mathrm{k}-\mathrm{f}+\mathrm{h}$.

10) $\mathrm{Now}$

$$
\begin{aligned}
\log u_{m}=(n+1) & \log k+(m-n) \log (1-k)+\log \Gamma(m+I) \\
& -\log \Gamma(n+I)-\log \Gamma(m-n+I) .
\end{aligned}
$$

11) $\log \Gamma(n+1)=(1 / 2) \log 2 \pi-n+(n+1 / 2) \log n+0(1 / n)$ by $(1.6)$. $\log \Gamma(n+1)=(1 / 2) \log 2 \pi-(n / k)+f-h$

$$
+(m+1 / 2) \log (n / k) \quad\left(1+\frac{k(h-r)}{n}\right)+0\left(\frac{l}{m}\right)
$$


Chap. 4

Since $\frac{1}{m}=\frac{1}{n} \frac{k}{1+\frac{k}{n}(h-f)}$ and $1+\frac{k}{n}(n-f) \rightarrow I$ as $n \rightarrow \infty$

for all $h$ such that $|h| \leq n^{\zeta}$, it follows that $O\left(\frac{I}{m}\right)=0\left(\frac{I}{n}\right)$.

Therefore $\log \Gamma(m+1)$

$=(1 / 2) \log 2 \pi-(n / k)+f-h+(m+l / 2) \log n-(m+l / 2) \log k$

$+(h / k)-f+h+1 / 2) \log \left(1+\frac{k(h-f)}{n}\right)+o\left(\frac{1}{n}\right)$

$=(1 / 2) \log 2 \pi-(n / k)+f-h+(m+1 / 2)(\log n-\log k)$

$+(h / k)-f+h+1 / 2)\left\{\frac{k}{n}(h-f)-\frac{k^{2}}{2 n^{2}}(h-f)^{2}+0\left(\frac{h^{3}}{n^{3}}\right)\right\}$

$+o\left(\frac{1}{n}\right) \quad$ by $(1.9)$

$=(1 / 2) \log 2 \pi-(n / k)+(m+1 / 2)(\log n-\log k)$

$+\frac{k}{2 n}(h-f)+\frac{k}{2 n}(h-f)^{2}-\frac{k^{2}}{4 n^{2}}(h-f)^{2}\{l+2(h-f)\}$

$+0\left(\frac{|n|^{3}}{n^{2}}\right)+0\left(\frac{|n|^{3}}{n^{3}}\right)+0\left(\frac{|n|^{4}}{n^{3}}\right)+0\left(\frac{1}{n}\right)$

Now $0\left(\frac{|h|^{3}}{n^{3}}\right)=0\left(\frac{|h|+1}{n}\right)$ and $0\left(\frac{|n|^{4}}{n^{3}}\right)=0\left(\frac{|n|^{3}}{n^{2}}\right)$.

Also $\frac{k}{2 n}(h-f)=0\left(\frac{|h|+1}{n}\right)$, $\frac{k}{2 n}(h-f)^{2}=\frac{k h^{2}}{2 n}+0\left(\frac{|h|+1}{n}\right)$, and

$$
\frac{k^{2}}{4 n^{2}}(n-n)^{2}(1+2(n-f))=0\left(\frac{|n|+1}{n}\right)+0\left(\frac{\left.\ln \right|^{3}}{n^{2}}\right)
$$


Chap. 4

Thus

12) $\log \left[(m+1)=(1 / 2) \log 2 \pi-\frac{n}{k}+(m+1 / 2)(\log n-\log k)\right.$

$$
+\frac{k h^{2}}{2 n}+0\left(\frac{|h|+I}{n}\right)+0\left(\frac{|h|^{3}}{n^{2}}\right) .
$$

Similarly,

$$
\begin{aligned}
& \log \Gamma(m-n+1)=(1 / 2) \log 2 \pi-\frac{n}{k}+n+f-n \\
& +(m-n+1 / 2) \log \left\{\left(\frac{n(1-k)}{k}\right)\left(1+\frac{k}{1-k} \frac{n-f}{n}\right)\right\}+0\left(\frac{1}{m-n}\right) .
\end{aligned}
$$

Since $\frac{1}{m-n}=\frac{1}{n}\left(\frac{1}{\frac{1-k}{k}+\frac{h-f}{n}}\right)$ and $\frac{1}{\frac{1-k}{k}+\frac{h-f}{n}}+\frac{k}{1-k}-$

as $n \rightarrow \infty$ for all h such that $|h| \leq n^{5}$,

$0\left(\frac{1}{m-n}\right)=0\left(\frac{1}{n}\right)$.

Therefore

$$
\begin{aligned}
& \log \Gamma(m-n+I)=(1 / 2) \log 2 \pi-\frac{n}{k}+n+f-h \\
& +(m-n+1 / 2)\{\log n-\log k+\log (1-k)\} \\
& +\left\{\frac{n(1-k)}{k}+h-f+1 / 2\right\}\left(\frac{k}{1-k} \frac{h-f}{n}-\frac{k^{2}}{2(1-k)^{2}} \frac{(h-f)^{2}}{n^{2}}+0\left(\frac{|h|^{3}}{n^{3}}\right)\right) \\
& +o\left(\frac{l}{n}\right) \text { by }(1.9) .
\end{aligned}
$$

Thus

$$
\begin{aligned}
& \log \Gamma(m-n+1)=(1 / 2) \log 2 \pi-\frac{n}{k}+n \\
& +(m-n+1 / 2)(\log n-\log k+\log (1-k)) \\
& +\frac{k n^{2}}{2(1-k) n}+0\left(\frac{\lfloor n+1}{n}\right)+0\left(\frac{\left.\ln \right|^{3}}{n^{2}}\right) .
\end{aligned}
$$


Chap. 4

Combining $(4.10),(4.11),(4.12)$ and $(4.13)$, we obtain $\log u_{m}=-(1 / 2) \log 2 \pi-(1 / 2) \log n+\log k-(1 / 2) \log (1-k)$

$-\frac{k^{2} h^{2}}{2(1-k) n}+0\left(\frac{|h|+1}{n}\right)+0\left(\frac{|h|^{3}}{n^{2}}\right)$.

Thus

$$
\begin{aligned}
& u_{m}=\frac{k}{\sqrt{2 \pi n(1-k)}} e^{-k^{2} h^{2} / 2(1-k) n}\left\{1+0\left(\frac{|h|+1}{n}\right)+0\left(\frac{|h|^{3}}{n^{2}}\right)\right\} \\
& \text { by }(1.8) \\
&=\sqrt{\frac{c}{\pi(n / k)}} e^{-\frac{c h^{2}}{n / k}}\left\{1+0\left(\frac{|h|+1}{n}\right)+0\left(\frac{|h|^{3}}{n^{2}}\right)\right\} .
\end{aligned}
$$

Now $\left|\frac{1}{\sqrt{n / k}}-\frac{1}{\sqrt{M}}\right|=\left|\frac{\sqrt{M}-\sqrt{n / k}}{\sqrt{n / k} \sqrt{M}}\right|=\left|\frac{\sqrt{M}-\sqrt{n / k}}{\sqrt{n / k} \sqrt{M}}\right| \frac{\sqrt{M}+\sqrt{n / k}}{\sqrt{M}+\sqrt{n / k}} \mid$

$$
=\frac{f}{\sqrt{n / k} M+(n / k) \sqrt{M}} \leq \frac{f}{2 M^{3 / 2}}=0\left(n^{-3 / 2}\right) .
$$

Thus

$$
\begin{aligned}
& (n / k)^{-1 / 2}=M^{-1 / 2}+0\left(n^{-3 / 2}\right) \\
& \text { Also since }\left|\frac{c h^{2}}{M}-\frac{c h^{2}}{n / k}\right|=\frac{c h^{2} f}{M(n / k)}=0\left(h^{2} / n^{2}\right) \\
& e^{-\frac{c h^{2}}{n / k}}=e^{-c h^{2} / M} e^{\left(\frac{c h^{2}}{M}-\frac{c h^{2}}{n / k}\right)}=e^{-c h^{2} / M} e^{0\left(n^{2} / n^{2}\right)} \\
& =e^{-c h^{2} / M}\left(1+0\left(n^{2} / n^{2}\right)\right) \text { by (1.8). }
\end{aligned}
$$


Snap. 4

54

Therefore

$$
\begin{aligned}
& \sqrt{\frac{c}{\pi n / k}} e^{-\frac{c h^{2}}{n / k}} \\
& =\sqrt{c / \pi M} e^{-c h^{2} / M}\left\{1+0\left(\frac{1}{n^{3 / 2}}\right)+0\left(h^{2} / n^{2}\right)+0\left(\frac{h^{2}}{n^{7 / 2}}\right)\right\} \\
& =\sqrt{c / \pi M} e^{-c h^{2} / M}\left\{1+0\left(\frac{|h|+1}{n}\right)\right\} .
\end{aligned}
$$

Thus we obtain:

$$
\text { (4.7) } \begin{aligned}
u_{m} & =\sqrt{c / \pi M} e^{-\operatorname{ch}^{2} / M}\left\{1+0\left(\frac{|h|+1}{n}\right)+0\left(\frac{|h|^{3}}{n^{2}}\right)\right\} \\
& =\sqrt{c / \pi M} e^{-\operatorname{ch}^{2} M}\left\{1+0\left(\frac{|h|+1}{M}\right)+0\left(\frac{|h|^{3}}{M^{2}}\right)\right\} \\
& \text { since } o\left(\frac{1}{n}\right)=0\left(\frac{1}{M}\right) .
\end{aligned}
$$

Thus part (4) is established.

Now $M_{2}=M-\left[n^{5}\right]$ and $M_{3}+\left[n^{5}\right]$. Since both $\frac{|n|+1}{n}$

and $\frac{\left.\ln \right|^{3}}{n^{2}}$ are $0(1)$ for $|h| \leq n^{\zeta}$, it follows from (4.14)

that both $u_{M_{2}}$ and $u_{M_{3}}$ are $0\left(n^{-1 / 2} e^{-\operatorname{ck}\left[n^{\zeta}\right]^{2} / n}\right)$.

Let $n^{\zeta}=\left[n^{\zeta}\right]+q$ where $0 \leq q<1$.

Then $\left[n^{\zeta}\right]^{2}=n^{2 \zeta}-2 q n^{\zeta}+q^{2}$, 
Chap. 4

55

and

$$
\begin{aligned}
e^{-c k\left[n^{\zeta}\right]^{2} / n=} & e^{-c k n^{2 \zeta-1}} e^{-c k\left(-2 q n^{\zeta}+q^{2}\right) / n} \\
= & 0\left(e^{-c k n^{2 \zeta-I}}\right) \\
& \text { since } e^{-c k\left(-2 q n^{\zeta}+q^{2}\right) / n}+1 \text { as } n \rightarrow \infty .
\end{aligned}
$$

Thus $u_{M_{2}}$ and $u_{M_{3}}$ are each $0\left(n^{-1 / 2} e^{-c k n} n^{2 \zeta-1}\right)$ and

thus $0\left(e^{-n^{n}}\right)$ for $0<n<2 \zeta-1$.

Thus parts (3) and (5) of the theorem are established and the theorem is proved.

(4.15) THEOREM.

$$
\begin{aligned}
& \quad I f a_{n}=o(I) \text { and } \sum_{n=0}^{\infty} a_{n}=A(\gamma, k) \text { then } \\
& A_{n}=o\left(n^{1 / 2}\right) .
\end{aligned}
$$

PROOF.

We use the same notation as earlier in this chapter.

$$
\begin{gathered}
\text { Since } \sum_{n=0}^{\infty} a_{n}=A(\gamma, k), B_{n}=A+o(1) \text { as } n \rightarrow \infty . \\
B_{n}=k^{n+1} \sum_{m=n}^{\infty}\left({ }_{n}^{m}\right)(1-k)^{m-n} A_{m}=\sum_{m=n}^{\infty} u_{m}(n) A_{m} \\
=\sum_{n=n-M}^{\infty} u_{M+h} A_{M+h}=\sum_{n=-\infty}^{\infty} u_{M+n} A_{M+h} .
\end{gathered}
$$


Chap. 4

56

Since $\sum_{h=-\infty}^{\infty} u_{M+h}=\sum_{m=n}^{\infty} u_{m}(n)=1$ by (4.6)

$$
\begin{aligned}
& \text { (4.16) } \sum_{h=-\infty}^{\infty} u_{M+h}\left(A_{M+h}-A_{M}\right)=B_{n}-A_{M}=A+o(I)-A_{M} \\
& \text { Since } a_{n}=o(I) \text { it follows that } A_{n}=o(n) \text { and } \\
& \text { thus } A_{n}=O(n) .
\end{aligned}
$$

Therefore

$$
\begin{aligned}
& |h|<n=u_{M+h}\left(A_{M+h}-A_{M}\right) \\
& =0\left(\sum_{|h|>n} \zeta^{(M+h)} u_{M+h}\right)+o\left(M \sum_{|h|>n} \sum^{u_{M+h}}\right) \\
& =0\left(e^{-n^{n}}\right)+0\left(n e^{-n^{n}}\right), \quad \text { by }(4.8) \text { with } \lambda=1 \text { and } \lambda=0 \text {, } \\
& =o(1) \text { as } n \rightarrow \infty \text { (or } M \rightarrow \infty) \text {. }
\end{aligned}
$$

Also

$$
\begin{aligned}
& |h| \leq n={ }^{u_{M+h}}\left(A_{M+h}-A_{M}\right) \\
& =\sum_{|n| \leq n} \tau_{M+n} \circ(|n|) \text { by (I.II) with } j=M \\
& =0\left(\sum_{|h| \leq n} \zeta u_{M+h}|h|\right) \text { since (I.II) holds uniformly } \\
& \text { for }|\mathrm{n}| \leq \mathrm{n}^{\zeta}
\end{aligned}
$$

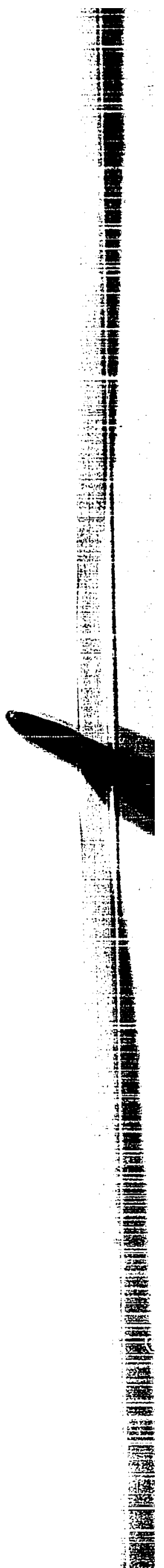


Chap. 4

$$
\begin{aligned}
& =0\left(\sum_{|h| \leq n} \zeta^{\sqrt{c / \pi M}}|h| e^{-\mathrm{ch}^{2} / M}\right) \text { by (4.7), since for } \\
& |h| \leq n^{5}, \frac{|n|+1}{n} \text { and } \frac{|n|^{3}}{n^{2}} \text { are each } O(1)
\end{aligned}
$$

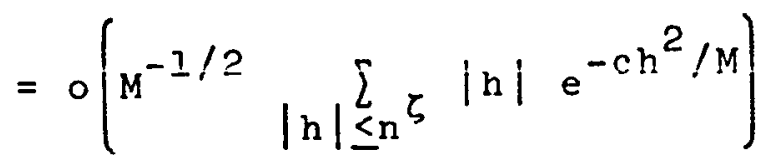

$$
\begin{aligned}
& =0\left(M^{-1 / 2} \int_{0}^{\infty} e^{-c t^{2} / M} t d t\right) \text { by }(2.7) \\
& =0\left(M^{-1 / 2}(M / c) \int_{0}^{\infty} e^{-u^{2}} u d u\right) \\
& =o\left(m^{1 / 2}\right) \text { as } M+\infty \text {. } \\
& \text { It. follows from }(4.16) \text { that }
\end{aligned}
$$$$
A+o(1)-A_{M}=o(1)+o\left(M^{1 / 2}\right)=o\left(M^{1 / 2}\right) \text {. }
$$

Therefore $A_{M}=o\left(M^{1 / 2}\right)$ and thus $A_{M}=o\left(n^{1 / 2}\right)$.

Since $a_{n}=o(1)$ and there are at most $[(1 / k)+1]$ terms between $[n / k]$ and $[(n+1) / k]$, it follows that $A_{n}=o\left(n^{1 / 2}\right)$.

(4.17) LEMMA.

$$
\text { If } \mathrm{A}_{\mathrm{n}}=\mathrm{o}\left(\mathrm{n}^{1 / 2}\right) \text { and } \mathrm{R} \text { is the radius of convergence }
$$

$$
\text { of } \sum_{n=0}^{\infty} a_{n} x^{n} \text { then } R \geq 1 \text {. }
$$


Chap. 4

58

PROOF.

$$
\begin{aligned}
& \text { Since } A_{n}=o\left(n^{1 / 2}\right) \text {, } \\
& \left|a_{n}\right|=\left|A_{n}-A_{n-1}\right| \leq\left|A_{n}\right|+\left|A_{n-1}\right|=o\left(n^{1 / 2}\right)+o\left(n^{1 / 2}\right) . \\
& \text { Therefore }\left|a_{n}\right|=o\left(n^{1 / 2}\right) \text {. } \\
& \text { Thus }\left|a_{n}\right| \leq n^{1 / 2} \text { for } n \geq N_{0} \text { and thus } \\
& \left|a_{n}\right|^{1 / n} \leq n^{1 / 2 n}=\sqrt{n^{1 / n}} \\
& \text { Therefore } \lim \sup _{n \rightarrow \infty}\left|a_{n}\right|^{1 / n} \leq \lim _{n \rightarrow \infty} \sup _{n} \sqrt{n^{1 / n}} \\
& =\lim _{n \rightarrow \infty} \sqrt{n^{1 / n}}=1 \text {. }
\end{aligned}
$$

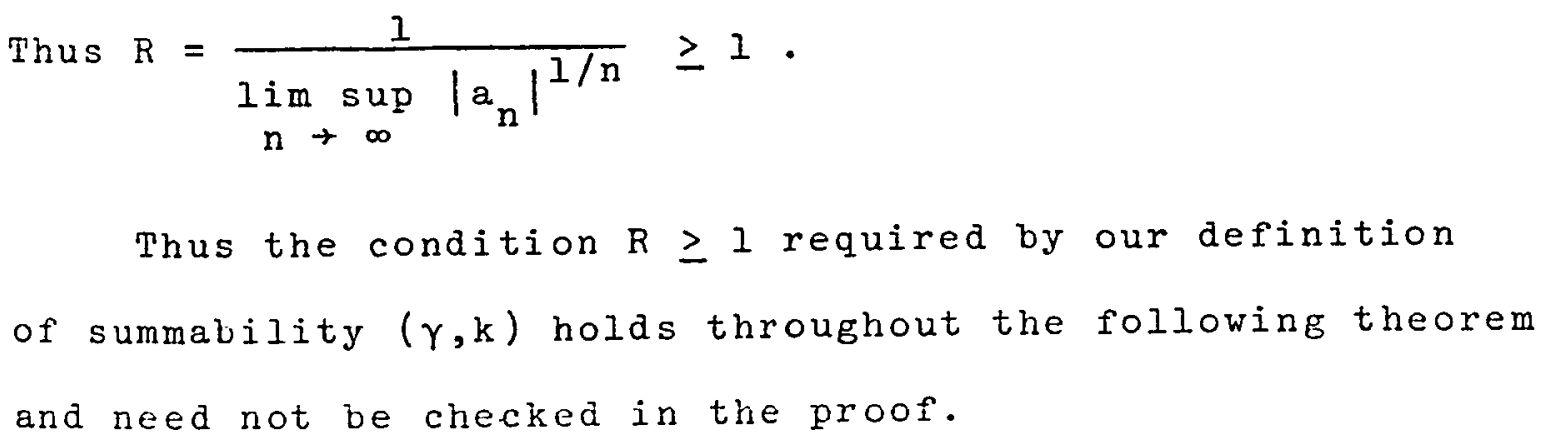

(4.18) THEOREM.

$$
\text { If } A_{n}=o\left(n^{1 / 2}\right) \text { then } \sum_{n=0}^{\infty} a_{n}=A(\gamma, k) \text { if and }
$$

only if $\sum_{n=0}^{\infty} a_{n}=A(e, c)$ where $c=\frac{k}{2(?-k)}$. 
Chap. 4

PROOF.

That we may assume $A=0$ without loss of generality here follows from the regularity of the methods in exactly the same way that it did in Theorem (3.8). We therefore take $A=0$. In the notation we have used throughout this chapter, we must show that

$$
\begin{aligned}
& \sum_{h=-\infty}^{\infty} u_{M+h} A_{M+h}=o(1) \text { as } n \rightarrow \infty \text { if and only if } \\
& \sqrt{c / \pi n} \sum_{h=-\infty}^{\infty} e^{-c h^{2} / n} A_{n+h}=o(1) \text { as } n \rightarrow \infty .
\end{aligned}
$$

Now

$$
\begin{aligned}
& |h| \sum_{n} \zeta u_{M+h} A_{M+h}=\sum_{|h|>n} \zeta u_{M+h} O(M+h) \quad \text { since } A_{n}=o(n) \\
& =0\left(\sum_{|h|>n} \zeta(M+h) u_{M+h}\right) \\
& =0\left(e^{-n}\right) \\
& =0(1) \text { as } n \rightarrow \infty .
\end{aligned}
$$

Therefore

(4.19) $\sum_{|h|>n} \zeta u_{M+h} A_{M+h}=o(1)$ as $n+\infty$. 
Chap. 4

60

Also

$$
\begin{aligned}
& |h| \leq n^{5} u_{n+h} A_{M+h}
\end{aligned}
$$

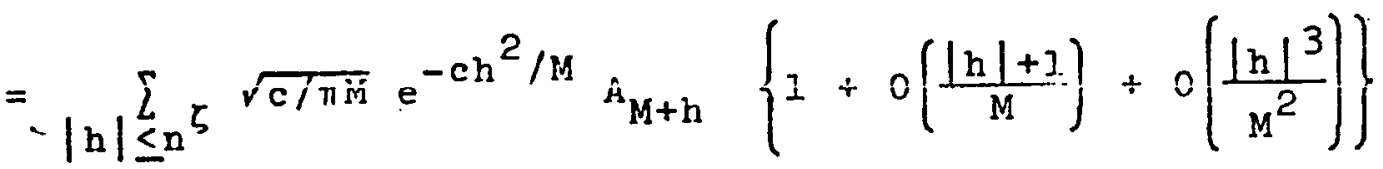

$$
\begin{aligned}
& \text { by }(4.7) \\
& =\sqrt{c / \pi M} \sum_{|h| \leq n} S^{e^{-c h^{2} / M} A_{M+h}+S+T}
\end{aligned}
$$

where

$$
\begin{aligned}
& S=\sqrt{c / \pi M}|h| \leq n \zeta e^{-c h^{2} / M} A_{M+h} 0\left(\frac{|h|+1}{M}\right) \\
& =\sqrt{c / \pi M}|\ln | \leq n \zeta{ }^{-c h^{2} / M} \circ\left(M^{I / 2}\right) \circ\left(\frac{|h|+1}{M}\right) \\
& \text { by (1.12) with } j=M \\
& =o\left\{M^{-1}|h| \leq n \zeta^{-c h^{2} / M}(|h|+1)\right\} \\
& \text { since (1.12) holds uniformly for }|h| \leq n^{\zeta} \\
& =0\left(M^{-1} \int_{0}^{\infty} e^{-c t^{2} / M}(t+1) d t\right) \text { by (2.5) and }(2.7) \\
& =0\left(M^{-1}(\sqrt{M / 2 c}+\sqrt{M / c} \cdot \sqrt{\pi / 2})\right) \\
& =0(1) \div 0\left(M^{-1 / 2}\right) \\
& =o(I) \text { as } H \rightarrow \infty \text {, } \\
& \text { and } \\
& T=\sqrt{c / \pi M} \sum_{|n| \sum_{i s} \zeta} e^{-\operatorname{ch}^{2} / M} \Lambda_{M+h} \circ\left(\frac{|h|^{3}}{M^{2}}\right)
\end{aligned}
$$




$$
\begin{aligned}
& =0\left\{M^{-2}|\ln | \leq n \zeta e^{-c h^{2} / M}|h|^{3}\right) \quad \text { as for } s \text { above } \\
& =0\left\{M^{-2} \int_{0}^{\infty} e^{-c t^{2} / M} t^{3} d t\right) \quad \text { by }(2.10) \\
& =0\left\{M^{-2}(M / c)^{2}(1 / 2)\right\} \\
& =0(1) \text { as } M \rightarrow \infty .
\end{aligned}
$$

Thus

(4.20) $\sum_{|h| \leq n} \zeta^{u_{M+h} A_{M+h}}=\sqrt{c / \pi M} \sum_{|h| \leq n} \sum^{-c h^{2} / M} A_{M+h}+o(1)$.

Now

$$
\begin{aligned}
& \sqrt{c / \pi M} \sum_{|h|>n} \zeta^{e^{-c h_{1}^{2} / M} A_{M+h}} \\
& =\sqrt{c / \pi M} \sum_{|h|>n} \zeta^{-c h^{2} / M} o(|h|) \\
& \text { by (1.13) with } \alpha=1 \text { and } j=M \\
& =0\left(M^{-1 / 2} \sum_{|h|>n} \int^{-\operatorname{ch}^{2} / M}|h|\right) \\
& =0\left(M^{-1 / 2} \int_{n}^{\infty} e^{-c t^{2} / M} t d t\right) \quad \text { by }(2.8) \text { with } a=1 \\
& =0\left\{m^{-1 / 2}\left(-(M / 2 c) e^{-c t^{2} / M}\right]_{n}^{\infty} \zeta\right\} \\
& \left.=0\left\{M^{-1 / 2}(M / 2 c) e^{-c n^{2 \zeta} / M}\right)\right\}
\end{aligned}
$$


Chap. 4

$$
\begin{aligned}
& =0\left[y^{1 / 2} e^{-\frac{c n^{2}}{n / k-f}}\right) \text { where } M=[n / k]=n / k-f, 0 \leq f<1 \\
& =0\left(M^{1 / 2} e^{-c k n^{2 \zeta-1}}\right) \\
& \text { since } n / k-f<n / k \text { and therefore } \frac{-1}{n / k-f}<\frac{-1}{n / k} \\
& =0\left(n^{1 / 2} e^{-\rho n^{a}}\right) \text { where } \rho=c k>0 \text { and } a=2 \zeta-1>0 \\
& =0(1) \text { as } M \rightarrow \infty \text {. }
\end{aligned}
$$

Therefore

(4.21) $\sqrt{c / \pi M} \sum_{|h|>n} \zeta e^{-c h^{2} / M} \quad A_{M+h}=0(1)$ as $M+\infty$.

Thus

$$
\begin{aligned}
& \sum_{h=-\infty}^{\infty} u_{M+h} A_{M+h}=o(I) \text { if and only if } \\
& \ln \mid \sum_{\leq n} \zeta{ }^{u_{M+h}}{ }^{A_{M+h}}=o(1) \quad(b y(4.19)) \text {, if and only if } \\
& \sqrt{c / \pi M} \sum_{|n| \leq n} \int^{-c h^{2} / M} A_{M+h}=o(I) \quad(b y(4.20)) \text {, if and only if } \\
& \sqrt{c / \pi M} \sum_{h=-\infty}^{\infty} e^{-c h^{2} / M} A_{M+h}=o(1) \quad(\text { by }(4.21)) \text {, if and only if } \\
& \sqrt{c / \pi n} \sum_{n=-\infty}^{\infty} e^{-c h^{2} / n} A_{n+h}=o(I) \quad(\text { replacing } M \text { by } n) \text {. }
\end{aligned}
$$


Chap. 4

Thus

$$
\sum_{n=0}^{\infty} a_{n}=A(\gamma, k) \text { if and only if } \sum_{n=0}^{\infty} a_{n}=A\left(e, \frac{k}{2(1-k)}\right)
$$

and the theorem is proved.

(4.22) COROLLARY.

$$
\begin{aligned}
& \text { If } a_{n}=o(I) \text { then } \sum_{n=0}^{\infty} a_{n}=A(\gamma, k) \text { if and only } \\
& \text { if } \sum_{n=0}^{\infty} a_{n}=A(e, c) \text { where } c=\frac{k}{2(1-k)} \text {. } \\
& \text { When k increases from } 0 \text { to } 1, \text { c increases from } 0 \text { to } \infty . \\
& \text { Combining Theorems }(4.4) \text { and (4.18) we thus obtain: }
\end{aligned}
$$

\section{(4.23) THEOREM.}

$$
\begin{aligned}
& \quad I f A_{n}=o\left(n^{1 / 2}\right) \text { and } \sum_{n=0}^{\infty} a_{n}=A(e, c) \text { then for } \\
& 0<d<c, \sum_{n=0}^{\infty} a_{n}=A(e, d) .
\end{aligned}
$$

(4.24) COROLLARY.

$$
\begin{aligned}
& \quad \text { If } a_{n}=o(I) \text { and } \sum_{n=0}^{\infty} a_{n}=A(e, c) \text { then for } \\
& 0<d<c, \sum_{n=0}^{\infty} a_{n}=A(e, d) .
\end{aligned}
$$

PROOF.

$$
\text { Just comine Theoreins }(4.23) \text { and (3.1). }
$$




\section{CHAPTER 5}

\section{VITALI'S THEOREM}

In this chapter we state and prove a theorem due to Vitali which is of critical importance in the proof of the Tauberian Theorem in the next chapter (see Littlewood [11, p. 117] or Titchmarsh [13, p. 168]). Let $\mathrm{D}$ be a region ( $i . e$. an open connected subset) of the complex plane. The functions $f$ of a family of functions $F$ are said to be almost uniformly bounded in $D$ if for each compact subset $A$ contained in $D$, there exists an $M$ such that $|f(z)| \leq M$ for all $z \in A$ and for all $f \in F$. Similarly a sequence $\left\{f_{n}\right\}$ of functions $f_{n}(z)$ is said to converge almost uniformly to $f(z)$ in $D$ (and we write $\left.f_{n}(z) \rightarrow f(z)\right)$ if $\left\{f_{n}\right\}$ converges uniformly to $f$ on every compact subset of $D$. We shall call a family $F$ of functions in $D$ a normal family if every sequence $\left\{f_{n}\right\}$ of functions $f_{n} \in F$ contains an almost uniformly convergent subsequence. For these definitions see Saks and Zygmund [12] and AhIfors [1]. 
Chap. 5

(5.1) THEOREM. (vitali)

Let $\mathrm{D}$ be a region of the complex plane and suppose:

(i) $\left\{\phi_{n}\right\}$ is a sequence of functions analytic and almost uniformly bounded in $D$,

and

(ii) there exists a sequence $\left\{\mathrm{z}_{\mathrm{m}}\right\}$ (of distinct $\mathrm{z}$ 's)

in $\mathrm{D}$ with at least one limit point $\mathrm{z}_{\mathrm{o}} \in \mathrm{D}$ such

that $\lim _{n \rightarrow \infty} \phi_{n}\left(z_{m}\right)$ exists (necessarizy finite because

of (i), for $m=1,2,3, \ldots$.

Then there exists a function $\phi(\mathrm{z})$, analytic in $\mathrm{D}$, such

that $\phi_{\mathrm{n}}(\mathrm{z}) \neq \phi(\mathrm{z})$.

PROOF.

Condition (i) implies that the sequence $\left\{\phi_{n}\right\}$ is a

normal family. (This is the theorem of stieltjes - Osgood.

See Saks and Zygmund [12, p. 119] or Ahlfors [1, Theorem

12 on p. 216].) We first claim that $\left\{\phi_{n}\right\}$ converges pointwise

for all $z$ in $D$. If not there exists a $z^{*} \in D$ for which

$\left\{\phi_{n}\left(z^{*}\right)\right\}$ does not tend to a limit. Then we can find two

subsequences of the positive integers $\left\{n_{k}{ }^{\prime}\right\}$ and $\left\{n_{k}{ }^{\prime}\right\}$ through

which $\left\{\phi_{n}\left(z^{*}\right)\right\}$ tends respectively to two values differing

by $c \neq 0$. (Any bounded sequence has a convergent subsequence.

If every convergent subsequence converged to the same 
value $\left\{\phi_{n}\left(z^{*}\right)\right\}$ would converge.) Let

$f_{k}(z)=\phi_{n_{k}},(z)-\phi_{n_{k}} "(z)$. Then $\left\{f_{i k}\right\}$ is a sequence

of analytic and almost uniformly bounded functions

in D. Thus (again by the theorem of stieltjes - osgocd)

$\left\{f_{k}(z)\right\}$ is a normal family and hence has an almost uniformly convergent subsequence. Let $f(z)$ be the limit of this almost uniformly convergent subsequence.

Then $f(z)$ is analytic by a theorem of Weierstrass. (See Saks and Zygmund [12, p. 116] or Ahlfors [1, Theorem 1 on p. 174].) Since $\left\{\phi_{n}\left(z_{m}\right)\right\}$ converges as $n+\infty$ for $m=1,2,3 \ldots$ it follows that $f\left(z_{m}\right)=0$ for $m=1,2,3 \ldots$. But then it follows from the identity theorem for analytic functions that $f(z)$ is identically 0 in $D$. This is a contradiction of $f\left(z^{*}\right)=c$. We conclude that $\left\{\phi_{n}(z)\right\}$ converges pointwise for all $z \in D$. Call the limit function $\phi(z)$. We now claim that $\phi_{n}(z) \rightarrow \phi(z)$ in $D$. This follows from a well known result (see Saks and Zygmund [12, (3.4) on p. 52]) since $\left\{\phi_{n}\right\}$ is a normal family and the sequence converges pointwise to $\phi(z)$. Since, however, a proof is easy, we Eive one here. If our claim does not hold, there exists a compact set $A$ contained in $D$, an $\varepsilon>0$, an increasing sequence of indices $\left\{n_{k}\right\}$, and a sequence of 
of points $\left\{z_{\mathfrak{t}}\right\}$ in $A$ such that

$\left|\hat{\phi}_{n_{k}}\left(z_{k}\right)-\phi\left(z_{k}\right)\right| \geq \varepsilon$ for $k=1,2,3 \ldots$. Since $\left\{\phi_{n}\right\}$

is a normal family, the sequence $\left\{\phi_{n_{k}}\right\}$ has a subsequence $\left\{\phi_{n_{j}}\right\}$ which converges uniformly on $A$ to $\phi(z)$.

Thus there exists an integer $N$ such that if $J \geq N$ $\left|\phi_{n_{k}}(z)-\phi(z)\right|<\varepsilon \quad$ for all $z \in A$. But $\left|\phi_{n_{k}}\left(z_{k_{j}}\right)-\phi\left(z_{k_{j}}\right)\right| \geq \varepsilon$ for all positive integers $j \cdot$ This is a contradiction. Therefore $\phi_{n}(z) \rightarrow \phi(z)$ in $D$. It now follows from the theorem of Weierstrass appealed to earlier that $\phi(z)$ is analytic in $D$, and the theorem is proved. 
CHAPTER 6

the "O" tauberian theORem for SuMmability $(B, \alpha, \beta)$

In this chapter we prove our main Theorem (6.7).

For convenience and to emphasize its structure, the

proof is divided among three theorems.

(For an outline of similar proofs in the case of Borel

summability see Hardy [8, section 9.13].)

\section{(6.1) THEOREM.}

$$
\text { If } a_{n}=0\left(n^{-1 / 2}\right) \text { and } \sum_{n=0}^{\infty} a_{n}=A(B, \alpha, \beta)
$$

then $A_{n}=O(I)$.

PROOF.

Since

$$
a_{n}=0\left(n^{-1 / 2}\right)
$$

it follows that

$$
a_{n}=o(1)
$$

and thus

$$
A_{n}=o\left(n^{I / 2}\right) \text { by }(1.2) \text { with } \rho=0 \text {. }
$$

Therefore

$$
\sum_{i=0}^{\infty} a_{n}=A(e, c / 2) \text { by Theorem (3.8). }
$$


Chap. 6

Thus

$$
\sqrt{a / 2 \pi n} \sum_{h=-\infty}^{\infty} e^{-\alpha h^{2} / 2 n} A_{n+h}=A+o(I) \text { as } n \rightarrow \infty .
$$

Since

$$
\sqrt{\alpha / 2 \pi n} \sum_{h=-\infty}^{\infty} e^{-\alpha h^{2} / 2 n}=1+o(1) \text { as } n+\infty \text { by }(2.4) \text {, }
$$

it follows that

$$
\begin{aligned}
& A_{n}\{1+o(1)\} \\
& =\sqrt{\alpha / 2 \pi n} \sum_{h=-\infty}^{\infty} e^{-\alpha h^{2} / 2 n} A_{n} \\
& =\sqrt{\alpha / 2 \pi n} \sum_{h=-\infty}^{\infty} e^{-\alpha h^{2} / 2 n} A_{n+h} \\
& +\sqrt{\alpha / 2 \pi n} \sum_{h=-\infty}^{\infty} e^{-\alpha h^{2} / 2 n}\left(A_{n}-A_{n+h}\right) \\
& =A+o(1)+\sqrt{\alpha / 2 \pi n}|h|>\sum^{5} e^{-\alpha h^{2} / 2 n}\left(A_{n}-A_{n+h}\right) \\
& +\sqrt{\alpha / 2 \pi n} \sum_{|h| \leq n} e^{-\alpha h^{2} / 2 n}\left(A_{n}-A_{n+h}\right) \\
& \text { Since } a_{n}=0(I) \text { it follows that } A_{n}-A_{n+h}=0(|h|) \text {. }
\end{aligned}
$$


Chap. 6

Thus

$$
\begin{aligned}
& \sqrt{\alpha / 2 \pi n}|h|>n \zeta e^{-\alpha h^{2} / 2 n}\left(A_{n}-A_{n+h}\right) \\
= & 0\left\{n^{-1 / 2}|h|>n^{\zeta} e^{-\alpha h^{2} / 2 n}|h|\right\} \\
= & 0\left\{n^{-1 / 2} \int_{0}^{\infty} e^{-\alpha t^{2} / 2 n} t d t\right\} \quad \text { by (2.8) } \\
= & \left.0\left\{n^{-1 / 2}(n / \alpha) e^{-\alpha n^{2 \zeta} / 2 n}\right)\right\} \\
= & 0\left\{n^{1 / 2} e^{-\alpha n} 2 \zeta-1 / 2\right\} \\
= & 0(1) \text { as } n \rightarrow \infty \text { since } \alpha>0 \text { and } 2 \zeta-1>0 .
\end{aligned}
$$

That is:

(6.3) $\sqrt{\alpha / 2 \pi n} \sum_{|h|>n} e^{-\alpha h^{2} / 2 n}\left(A_{n}-A_{n+h}\right)=o(I)$ as $n \rightarrow \infty$.

$$
\text { Since } a_{n}=0\left(n^{-1 / 2}\right), \quad\left|A_{n+h}-A_{n}\right|=0\left(\frac{|h|}{\sqrt{n}}\right)
$$

for $|h| \leq n^{\zeta}$ by $(1.14)$. 
Chap. 6

Thus

$$
\begin{aligned}
& \sqrt{\alpha / 2 \pi n}|h| \leq n \zeta{ }^{-\alpha h^{2} / 2 n}\left(A_{n}-\Lambda_{n+h}\right) \\
= & 0\left\{(1 / n)|n| \leq n \zeta e^{-\alpha h^{2} / 2 n}|n|\right\} \\
= & 0\left\{(1 / n) \int_{0}^{\infty} e^{-\alpha t^{2} / 2 n} t a t\right\} \quad \text { by (2.7) } \\
= & 0\{(1 / n) \cdot(n / \alpha)\} \\
= & 0(1) .
\end{aligned}
$$

That is:

(6.4) $\sqrt{\alpha / 2 \pi n} \sum_{|h| \leq n} 5^{-\alpha h^{2} / 2 n}\left(A_{n}-A_{n+h}\right)=0(1)$.

It follows from $(6.2),(6.3)$ and $(6.4)$ that

$A_{n}\{1+o(1)\}=O(1)+O(1)+A+o(I)=0(1)$.

Therefore $A_{n}=O(1)$.

(6.5) THEOREM.

$$
\text { If } \sum_{n=0}^{\infty} a_{n}=A(B, \alpha, \beta) \text { and } A_{n}=O(I) \text { then }
$$

$\sum_{n=0}^{\infty} a_{n}=A(e, c)$ for all positive $c$.

PROOF.

$$
\text { Let } z=x+i y \in K(\text { the complex plane). }
$$


Choose $y_{0}>0$ and $0<x_{0}<\alpha / 2$.

Let

$$
\begin{aligned}
D & =\left\{z \in K: x>x_{0} \text { and }|y|<y_{0}\right\} \\
\phi_{n}(z) & =\sqrt{z / \pi n} \sum_{h=-\infty}^{\infty} e^{-z h^{2} / n} A_{n+h} \\
& =\sqrt{z / \pi n} \sum_{h=-n}^{\infty} e^{-z h^{2} / n} A_{n+h} .
\end{aligned}
$$

and

$$
q=q(n)=e^{-x_{0} / n}
$$

Then $0<q<1$ and since $A_{n}=0(1)$,

$$
\begin{aligned}
& \left|e^{-z h^{2} / n} A_{n+h}\right| \leq H\left|e^{-z h^{2} / n}\right| \quad \text { for some constant } H \\
& =H e^{-x h^{2} / n} \leq H\left(e^{\left.-x_{0} / n\right) h^{2}}=H q^{h^{2}} \text { for all } z \in D .\right.
\end{aligned}
$$

Since $\sum_{h=0}^{\infty} H q^{h^{2}}=H \sum_{h=0}^{\infty} q^{h^{2}} \leq H \sum_{h=0}^{\infty} q^{h}=\frac{H}{1-q}$

it therefore follows from the Weierstrass M-test that

$\sum_{n=-n}^{\infty} e^{-z h^{2} / n} A_{n+h}$ converges uniformly in $D$, and is thus

analytic in D. Since $\sqrt{z / \pi n}$ is analytic in $D, \phi_{n}(z)$

is analytic in D. $(n=1,2,3, \ldots$. 
Chap. 6

In $D$,

$|z|=\sqrt{x^{2}+y^{2}}=x \sqrt{1+(y / x)^{2}} \leq x \sqrt{1+\left(y_{0} / x_{0}\right)^{2}}$

Therefore

$\sqrt{|z| / n}=O(\sqrt{x / n})$ uniformly for all $z \in D$.

Thus

$$
\begin{aligned}
\left|\phi_{n}(z)\right| & =0\left((|z| / \pi n) \sum_{h=-\infty}^{\infty}\left|e^{-z h^{2} / n}\right|\right) \\
& =0\left(\sqrt{x / \pi n} \sum_{h=-\infty}^{\infty} e^{-x h^{2} / n}\right) \\
& =0\{1+0(\sqrt{x / n})\} \quad \text { by }(2.3) \\
& =0(1) \quad \text { if } x \text { is bounded above (say }
\end{aligned}
$$

Therefore $\left\{\phi_{n}(z)\right\}$ is almost uniformly bounded in $D$. Thus the sequence $\left\{\phi_{n}\right\}$ satisfies condition (i) of Theorem (5.1.) (Vitali's Theorem).

$$
\text { Since } A_{n}=O(1) \quad, \quad A_{n}=0\left(n^{1 / 2}\right)
$$

and thus

$$
\sum_{n=0}^{\infty} a_{n}=A(e, c / 2) \text { by Thcorem }(3.8) \text {. }
$$


Chap. 6

Therefore

$(6.6)$

$$
\sum_{n=0}^{\infty} a_{n}=A(e, c) \text { for } 0<c \leq c / 2 \text {, by Theorem }(4.22) \text {. }
$$

Thus

$$
\phi_{n}(c)+A \text { as } n+\infty \text { for } x_{0} \leq c \leq \alpha / 2 \text { in } D \text {. }
$$

Thus condition (ii) of Theorem (5.I) is satisfied and it follows from Theorem (5.1) that $\phi_{n}(z) \rightarrow A$ as $n+\infty$ for all $z \in D$. (Since $\phi(z)=A$ on the closed interval $x_{0} \leq x \leq \alpha / 2$ it follows from the identity theorem for analytic functions that $\phi(z)$ is identically equal to $A$ in $D$. )

In particular

$$
\sum_{n=0}^{\infty} a_{n}=A(e, c) \text { for } x_{0} \leq c<+\infty .
$$

Combining this with $(6.6)$ gives

$\sum_{n=0}^{\infty} a_{n}=A(e, c)$ for all $c>0$ and the theorem is proved. The next result is the desired Tauberian theorem.

(6.7) THEOREM.

$$
\text { If } \sum_{n=0}^{\infty} a_{n}=\Lambda(B, \alpha, \beta) \text { and } a_{n}=0\left(n^{-1 / 2}\right)
$$

$\operatorname{then} \sum_{n=0}^{\infty} a_{n}=A$. 
Chap. 6

PROOF.

It follows from Theorems $(6.1)$ and $(6.5)$ that

(6.8) $\sqrt{c / \pi n} \sum_{h=-\infty}^{\infty} e^{-c h^{2} / n} \dot{A}_{n+h}=\dot{A}+o(1)$ as $n \rightarrow \infty$

for all c > 0 .

Now since $a_{n}=0(I)$ it follows that $A_{n}-A_{n+h}=0(|h|)$,

and

(6.9) $\sqrt{c / \pi n} \sum_{|h|>n} e^{-c h^{2} / n}\left(A_{n}-A_{n+h}\right)=o(1)$ as $n \rightarrow \infty$

fexactly as in the proof of $(6.3)$ - just replace $\alpha / 2$ there by $c$ here).

Since for any fixed positive $c$

$\sqrt{c / \pi n} \sum_{n=-\infty}^{\infty} e^{-c h^{2} / n}=1+o(1)$ as $n+\infty$ by (2.4),

it follows that

$$
\begin{aligned}
& A_{n}\{1+o(1)\} \\
& =\sqrt{c / \pi n} \sum_{n=-\infty}^{\infty} e^{-c n^{2} / n}\left(A_{n}-A_{n+h}\right) \\
& \quad+\sqrt{c / \pi n} \sum_{h=-\infty}^{\infty} e^{-c h^{2} / n} A_{n+h}
\end{aligned}
$$


Chap. 6

$=\sqrt{c / \pi n}|h| \leq n \zeta{ } e^{-c h^{2} / n}\left(A_{n}-A_{n+h}\right)+o(I)+A+o(I)$ by $(6.8)$ and $(6.9)$

Thus

$$
\begin{aligned}
(6.10) \quad A_{n}-A & =\sqrt{c / \pi n}|h| \leq n^{5} e^{-c h^{2} / n}\left(A_{n}-A_{n+h}\right)+o(1) \\
& \text { since } A_{n} \cdot o(1)=o(1) \quad\left(\text { because } A_{n}=o(1)\right) \\
& \text { For }|h| \leq n^{5}, A_{n}-A_{n+h}=0\left(\frac{|h|}{\sqrt{n}}\right) \text { by }(1.14)
\end{aligned}
$$

and thus

$$
\left|A_{n}-A_{n+h}\right| \leq \frac{H|h|}{\sqrt{n}} \text { for some constant } H \text { and for }
$$

all $\mathrm{h}$ with $|\mathrm{h}| \leq \mathrm{n}^{5}$ and $\mathrm{n}=1,2,3, \ldots$.

Therefore

$$
\begin{aligned}
& |\sqrt{c / \pi n}| h\left|\leq n \zeta{ }^{-c h^{2} / n}\left(A_{n}-A_{n+h}\right)\right| \\
& \leq(H / n) \sqrt{c / \pi}|h| \leq n^{5} e^{-c h^{2} / n}|h| \\
& \leq(H / n) \sqrt{c / \pi} 2\left(\int_{0}^{\infty} e^{-c t^{2} / n} t d t+(n / 2 e c)^{1 / 2}\right) \text { by }(2.6) \\
& =(H / n) \sqrt{c / \pi}\left(2 \int_{0}^{\infty} e^{-c t^{2} / n} t d t+(2 n / e c)^{1 / 2}\right)
\end{aligned}
$$


Chap. 6

$$
\begin{aligned}
& \left.=(H / n) \sqrt{c / \pi}(n / c)+(2 n / \epsilon c)^{1 / 2}\right) \\
& =H / \sqrt{\pi c}+H \sqrt{2 / \pi e} n^{-1 / 2} \\
& =H / \sqrt{\pi c}+O(1) \text { as } n \rightarrow \infty .
\end{aligned}
$$

It follows from $(6.10)$ that

$$
\left|A_{n}-A\right| \leq H / \sqrt{\pi c}+O(I) \text {. }
$$

Thus

$$
\lim \sup \left|A_{n}-A\right| \leq \lim \sup _{n \rightarrow \infty}(H / \sqrt{\pi c}+o(1))=H / \sqrt{\pi c} .
$$

Since this holds for all positive $c$, it follows that

$$
0 \leq \underset{n \rightarrow \infty}{\lim \cdot \inf }\left|A_{n}-A\right| \leq \underset{n \rightarrow \infty}{\lim \sup }\left|A_{n}-A\right| \leq 0 .
$$

Thus

$$
\lim _{n \rightarrow \infty}\left|A_{n}-A\right|=0 \text { and } A_{n}+A \text { as } n \rightarrow \infty \text {. }
$$

Therefore

$$
\sum_{n=0}^{\infty} a_{n}=A \text { and the theorem is proved. }
$$

(6.11) COROLLARY.

$$
\begin{gathered}
\text { If } a_{n}=0\left(n^{-1 / 2}\right) \text { and either } \sum_{n=0}^{\infty} a_{n}=A(E, c) \\
\text { Or. } \sum_{n=0}^{\infty} a_{n}=\Lambda(\gamma, k) \text {, then } \sum_{n=0}^{\infty} a_{n}=A .
\end{gathered}
$$


Chap. 6

PROOF :

$$
\text { Just combine Theorems }(3.1),(3.8),(4.18) \text {, and }
$$
$(6.7)$. 


\section{REFERENCES}

[1] L. V. Ahlfors,

"Complex Analysis", (2nd edition),

McGraw - Hill,

(1966).

[2] D. Borwein,

"On Borel-type methods of summability",

Mathematika,

5; (1958), $128-133$.

[3] D. Borwein,

"Relations between Borel-type methods of

summability",

Journal London Math. Soc.,

35, $(1960), 65-70$.

[4] D. Borwein,

"On methods of summability based on

integral functions II",

Proc. Cambriage Phiz. Soc.,

$56,(1960), 125-131$. 
[5] D. Borwein,

"A Tauberian theorem for Borel-type methods of summability", Canadian Journal of Math., 21, $(1969), 740-747$.

[6] I. I. Good,

"Relations between methods of summation of series",

Proc. Camb. Phiz. Soc., $38(1942), 144-165$.

[7] G. H. Hardy,

"On the summability of series by Borel's and Mittag-Leffler's methods", Journal London Math. Soc., $9(1934), 153-157$.

[8] G. $\underline{H} \cdot \underline{H a r d y}$,

"Divergent Series", Oxford, (1949). 
[9] G. H. Hardy, and J. E. Littlewood,

"Theorems concerning the summability of series by Borel's exponential method", Rendiconti del Circolo matematico di Palermo, $41,(1916), 36-53$.

[10] Bateman Manuscript Project,

"Higher transcendental functions", vol. I, McGraw - Hill, (1953)

[11] J. E. Littlewood,

"Lectures on the theory of functions", oxfor $d$, $(1943)$

[12] S. Saks, and $\underline{A}$. Zygmund, "Analytic functions", Monogrefie Matematyczne Warszara, $(1952)$ 
[13] E. C. Titchmarsh,

"The theory of functions", (2nd edition),

Oxford,

(1939).

[14] L. Wzodarski,

"Propriétés des méthodes continues de limitation du type de Borel",

Buzz. Acad. Polon. Sci.,

CI. III, $4(1956), 173-175$. 